\title{
Beryllium-specific CD4+ $T$ cells induced by chemokine neoantigens perpetuate inflammation
}

\author{
Michael T. Falta, ${ }^{1}$ Jeremy C. Crawford, ${ }^{2}$ Alex N. Tinega, ${ }^{1}$ Laurie G. Landry, ${ }^{3}$ Frances Crawford, ${ }^{4}$ Douglas G. Mack, ${ }^{1}$ Allison K. Martin, ${ }^{1}$ \\ Shaikh M. Atif, ${ }^{1}$ Li Li, ${ }^{1,5}$ Radleigh G. Santos, ${ }^{6}$ Maki Nakayama, ${ }^{3,7}$ John W. Kappler, ${ }^{4,7}$ Lisa A. Maier, ${ }^{1,5}$ Paul C. Thomas, ${ }^{2}$ \\ Clemencia Pinilla, ${ }^{8}$ and Andrew P. Fontenot ${ }^{1,7}$ \\ 'Department of Medicine, University of Colorado Anschutz Medical Campus, Aurora, Colorado, USA. ²Department of Immunology, St. Jude Children's Research Hospital, Memphis, Tennessee, USA. \\ ${ }^{3}$ Barbara Davis Center for Childhood Diabetes, University of Colorado Anschutz Medical Campus, Aurora, Colorado, USA. ${ }^{4}$ Department of Biomedical Research and ${ }^{5}$ Department of Medicine, National Jewish \\ Health, Denver, Colorado, USA. 'Department of Mathematics, Nova Southeastern University, Ft. Lauderdale, Florida, USA. Department of Immunology and Microbiology, University of Colorado Anschutz \\ Medical Campus, Aurora, Colorado, USA. ${ }^{8}$ Florida International University, Port St. Lucie, Florida, USA.
}

Discovering dominant epitopes for T cells, particularly CD4+ $\mathrm{T}$ cells, in human immune-mediated diseases remains a significant challenge. Here, we used bronchoalveolar lavage (BAL) cells from HLA-DP2-expressing patients with chronic beryllium disease (CBD), a debilitating granulomatous lung disorder characterized by accumulations of beryllium-specific (Be-specific) $\mathrm{CD}^{+} \mathrm{T}$ cells in the lung. We discovered lung-resident $\mathrm{CD4} 4^{+} \mathrm{T}$ cells that expressed a disease-specific public CDR3 $\beta$ T cell receptor motif and were specific to Be-modified self-peptides derived from C-C motif ligand 4 (CCL4) and CCL3. HLADP2-CCL/Be tetramer staining confirmed that these chemokine-derived peptides represented major antigenic targets in CBD. Furthermore, Be induced CCL3 and CCL4 secretion in the lungs of mice and humans. In a murine model of CBD, the addition of LPS to Be oxide exposure enhanced CCL4 and CCL3 secretion in the lung and significantly increased the number and percentage of $\mathrm{CD}^{+} \mathrm{T}$ cells specific for the HLA-DP2-CCL/Be epitope. Thus, we demonstrate a direct link between Be-induced innate production of chemokines and the development of a robust adaptive immune response to those same chemokines presented as Be-modified self-peptides, creating a cycle of innate and adaptive immune activation.

\section{Introduction}

Identification of antigens activating $\mathrm{T}$ cells in immune-mediated diseases remains an elusive goal, yet offers the potential of providing insights into disease mechanisms and targets for antigen-specific therapies. The ongoing challenge requires integrating data from each component of the trimolecular complex: the presenting MHC molecule that may have to be inferred from genetic association studies (1), the peptide bound by the MHC, and the responding $\alpha \beta T C R$ expressed on the surface of disease-relevant $T$ cells. In this regard, the size of the immunopeptidome is considerable $(2,3)$, subject to posttranslational modifications, and understanding of its complexity is exacerbated by recent data indicating that fusion peptides make up a significant fraction of MHC-binding peptides (4-6). In addition, the postthymic TCR repertoire is enormous (7), and choosing candidate T cells from bystanders in the target organ based on TCR expression is problematic. For example, the molecular rules for assessing whether distinct TCRs share a common MHC/peptide specificity have been, until recently, ill defined. Finally, for all $\mathrm{T}$ cells under consideration for unbiased

Related Commentary: https://doi.org/10.1172/JCI148674

Conflict of interest: The authors have declared that no conflict of interest exists. Copyright: (5) 2021, American Society for Clinical Investigation.

Submitted: October 2, 2020; Accepted: February 23, 2021; Published: May 3, 2021.

Reference information: J Clin Invest. 2021;131(9):e144864.

https://doi.org/10.1172/JCl144864 antigen-specificity studies, obtaining paired TCR $\alpha$ - and $\beta$-chain sequences is a nontrivial task.

In the current study, we isolated bronchoalveolar lavage (BAL) T cells from patients with chronic beryllium disease (CBD), a granulomatous lung disorder that is triggered in susceptible individuals by inhalation of beryllium-containing (Be-containing) particulates. $\mathrm{CBD}$ is characterized by a well-defined genetic association of HLA-DPB1 alleles containing a glutamic acid (E) at the 69th position of the $\beta$-chain ( $\beta$ Glu69) (reviewed in ref. 8 ) and a robust $\mathrm{CD}^{+}{ }^{+} \mathrm{T}$ cell alveolitis (9). A substantial proportion of those $\mathrm{T}$ cells recognize HLA-DP-self-peptide/Be complexes, in which the addition of Be alters the topology and charge of the complex and generates neoantigens that are absent in the thymus and no longer tolerated by the immune system (10-12). Thus, CBD represents a potentially novel autoimmune disease with large numbers of neoantigen-specific T cells available from the lung, enabling an investigation of dominant $\mathrm{T}$ cell epitopes in a target organ.

To determine major epitopes recognized by lung-resident $\mathrm{CD}^{+} \mathrm{T}$ cells in CBD, we utilized single-cell sorting and reverse transcription-PCR (RT-PCR) amplification of TRA and TRB genes and determined complete $\alpha \beta T C R s$ from substantial numbers of $\mathrm{CD}^{+} \mathrm{T}$ cells derived from the BAL of CBD patients. These TCRs were evaluated using TCR distance measurements (13) to objectively predict those cells likely to share epitope specificity. Notably, we identified a public TCR repertoire in CBD patients expressing $H L A-D P B 1^{*} 02: 01$ (the most prevalent $\beta G l u 69$-containing allele) and determined that these $\mathrm{T}$ cells share specificity 
to related peptides derived from C-C motif ligand 3 (CCL3) and CCL4 presented by HLA-DP2 in the presence of Be. HLA-DP2$\mathrm{CCL} / \mathrm{Be}$ tetramer staining showed these ligands were recognized by large fractions of $\mathrm{CD}^{+} \mathrm{T}$ cells in the BAL of patients and HLADP2 Tg mice exposed to Be. These chemokines were also elevated in the lungs of CBD patients and Be-exposed HLA-DP2 Tg mice, and their expression was induced by exposure to Be. Further, the addition of a single dose of LPS to Be oxide (BeO) exposure in HLA-DP2 Tg mice enhanced CCL4 and CCL3 secretion in the lung and markedly increased the frequency of $\mathrm{CD} 4^{+} \mathrm{T}$ cells specific for the HLA-DP2-CCL/Be neoantigen. Thus, our data demonstrate a direct link between the innate immune mediators CCL3 and CCL 4 that are secreted in the lung in response to $\mathrm{Be}$ and a subsequent autoreactive $\mathrm{CD} 4^{+} \mathrm{T}$ cell response targeting these same molecules modified by Be, creating a positive feedback loop of persistent innate and adaptive immune activation.

\section{Results}

Single-cell analysis of TR genes expressed by BAL CD4 $4^{+} T$ cells. To identify antigenic targets of T cells in CBD, we examined TCRs expressed on $\mathrm{CD}^{+} \mathrm{T}$ cells in the lung of 7 HLA-DP2-expressing $\mathrm{CBD}$ patients. Individually sorted $\mathrm{CD} 4^{+} \mathrm{T}$ cells were sequenced for paired TRA and TRB gene expression from BAL T cell lines from 3 CBD patients (total $307 \alpha \beta$ TCRs obtained) and directly from ex vivo BAL samples from 4 additional patients (total $336 \alpha \beta$ TCRs). Assessing the clonal distribution of TCRs among these patients, there was increased clonality and reduced diversity in the $\mathrm{T}$ cell lines, though each individual still demonstrated a range of $\mathrm{T}$ cells expressing unique $\alpha \beta$ TCRs $(20 \%-60 \%)$ and no single TCR represented a majority of the culture (Figure 1A). The TCR repertoire of ex vivo BAL CD4 $4^{+} \mathrm{T}$ cells was substantially more diverse, with more than $60 \%$ of the cells expressing unique $\alpha \beta T C R$ pairs and smaller clonal expansions evident in the remaining cells (Figure 1A). This difference was also reflected in the inverse Simpson's diversity of clone-size distributions, where the 4 ex vivo samples were significantly more diverse than the $3 \mathrm{~T}$ cell lines (Figure 1B).

TCR gene-usage patterns were examined using a cord diagram for each patient's unique clonotypes and for all clonotypes combined; however, no obvious dominant gene segment usage was seen (Figure 1C, Supplemental Figure 1; supplemental material available online with this article; https://doi.org/10.1172/ JCI144864DS1). Therefore, we applied the TCRdist algorithm, a similarity measure that focuses on TCR contact residues with MHC-peptide complex to identify TCRs likely to share specificity, to the pooled clonotype repertoire across patients and plotted each clonotype as a principal components analysis (PCA) in TCRdist space (Figure 1D). This approach captures clusters of closely related TCRs in sequence space, but no apparent clusters were found. Thus, at the level of summary statistics or searching for shared sequence clusters, these data indicated that the lung-resident $\mathrm{T}$ cell repertoire is diverse and not dominated by an obvious group of clonally related TCRs.

Due to the lack of clear receptor associations, we used a rigorous motif-searching tool within the TCRdist algorithm to identify sequences enriched within the CBD TCR repertoires. This search compared the CBD TCR sequences to a background naive repertoire that represents the distribution of generation prob- abilities for TCRs in humans. The search also highlights amino acid residues within motifs unlikely to be generated by germline recombination, but rather required nucleotide deletion or $\mathrm{N}$ - or P-nucleotide addition. Using this search, we identified a highly statistically significant TCR CDR $\beta$ motif, CASSLKGGG, that occurred in 6 subjects (Figure 1E). Within this motif, the lysine residue was entirely non-germline derived (Figure $1 \mathrm{E}$ ), and the glycine triplet (GGG) sometimes varied at 1 or 2 positions by encoding an alanine, serine, or leucine. Cells expressing this motif were slightly enriched for TRBV27 gene usage, but other $T R B V$ genes, including $T R B V 7-2,7-3$, and 5-5, were used. TRB gene-segment usage and deduced CDR3 amino acid sequences for all $\mathrm{T}$ cells expressing the CDR3 $\beta$ motif are displayed in Figure $1 F$. In addition, these data emphasize how no other TCR components (e.g., TRAV, TRAJ, TRBJ, CDR3 $\alpha$, and junctional lengths) were conserved among these $\mathrm{T}$ cells (Figure $1 \mathrm{~F}$ and Supplemental Table 1). Thus, in contrast to antigen-specific $\mathrm{T}$ cells defined in other pathogen-specific responses that rely on both $\mathrm{V}$ and $\mathrm{J}$ gene usage enrichment and unique CDR3 features, we identified a CBD-associated motif characterized primarily by specific nongermline residues (LK) within an otherwise diverse repertoire.

In order to determine whether T cells expressing the CDR3 $\beta$ motif were Be specific, hybridoma cell lines were constructed to express human CD4 and a subset of these TCRs (Figure 1F) and TCR-expressing hybridomas were sorted for high TCR expression (data not shown). As shown in Figure 1G, these hybridomas released large quantities of IL-2 in response to $\mathrm{BeSO}_{4}$ presented by splenic B cells from HLA-DP2 Tg mice. These activation assays relied on the antigen-presenting cells to inherently provide the endogenous (unknown) peptide to complete the Be-dependent ligand. No other exogenous protein or peptide was added to the culture. Furthermore, activation of the LKGGG CDR3 $\beta$ TCRs was strictly dependent on the presence of Be. Addition of other metal cations, including $\mathrm{NiSO}_{4}, \mathrm{AlSO}_{4}$, or supplementing the medium with additional $\mathrm{CaCl}_{2}$ or $\mathrm{MgSO}_{4}$, failed to induce IL-2 secretion in 4 of the $\mathrm{T}$ cell hybridomas (Supplemental Figure 2A). Finally, the Be-specific response was HLA-DP2 dependent, as other HLA-DP molecules separately expressed on fibroblast cell lines failed to activate these TCRs in the presence of $\mathrm{BeSO}_{4}$ (Supplemental Figure 2B).

Mimotopes completing the ligand for Be-specific LKGGG CDR3 $\beta$ T cell receptors. T cell hybridomas expressing the 8133-c4r and 8845c3 TCRs were chosen as representatives of the LKGGG CDR3 $\beta$ public repertoire to assess peptides that complete the Be-dependent ligand. We screened an unbiased synthetic combinatorial decapeptide positional scanning library (PSL) (200 mixtures; refs. $14,15)$ using HLA-DP2-transfected kidney fibroblasts (DP2.21) as antigen-presenting cells in assays containing low concentrations of FBS (0.5\%). This cell line was derived from C57BL/6 mice deficient for the murine MHCII and invariant chain genes (11) and is unable to naturally present $\mathrm{Be}$ to Be-specific hybridomas when cultured in serum-free or -low conditions (11). Hybridoma IL-2 responses to all peptide mixtures in the presence of $\mathrm{BeSO}_{4}$ are shown in Supplemental Figure 3. As depicted in Figure 2A, both TCRs displayed preferences for acidic amino acids at peptide positions p4 and p5 (aspartic acid) and positions p7 and p8 (glutamic acid). To resolve whether a stimulatory peptide for these hybridomas might be composed of D4D5 and E7E8 doublets, we synthe- 
A

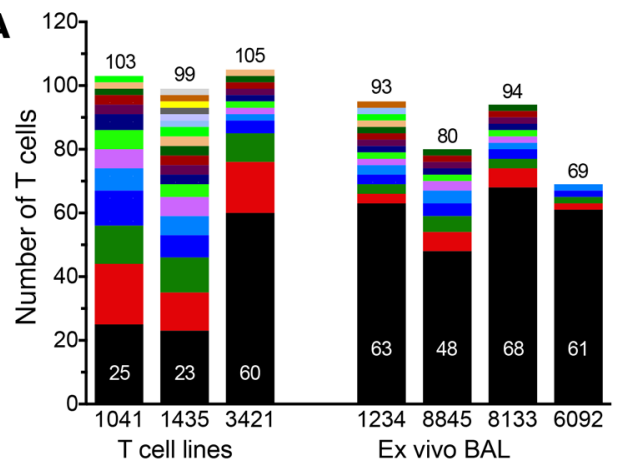

C

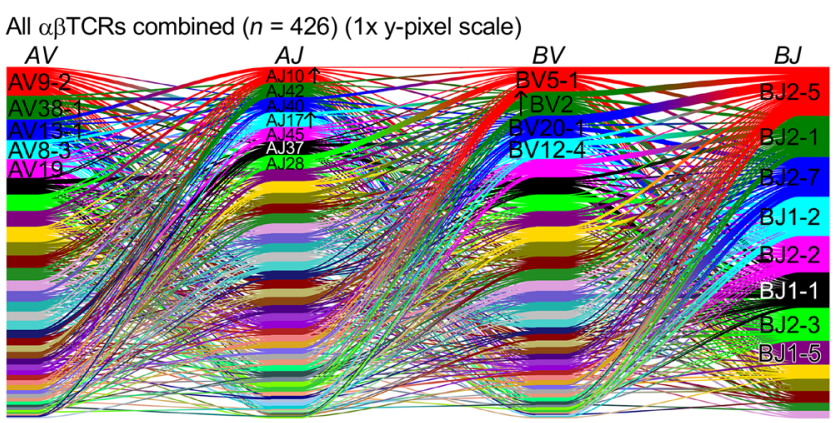

B

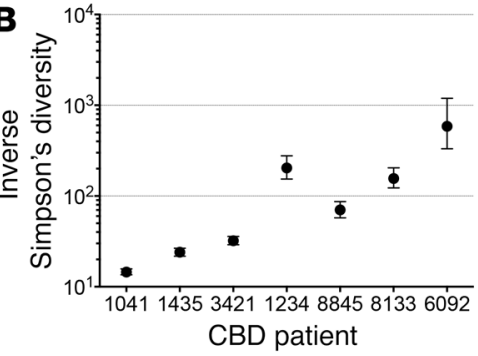

D

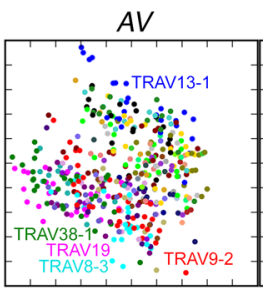

AJ

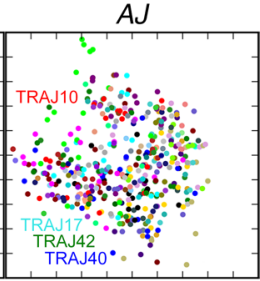

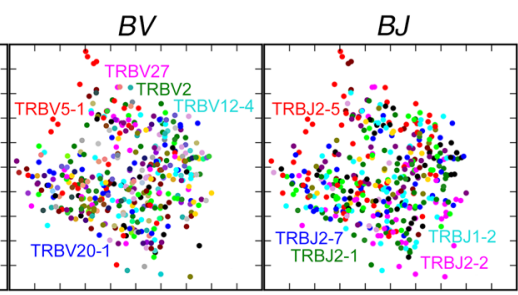

E

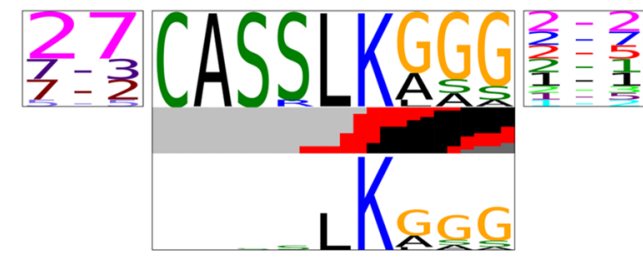

No. of total $\alpha \beta$ TCRs obtained: $\quad 643$

No. of unique $\alpha \beta T C R s: \quad 426$

No. of $\alpha \beta$ TCRs comprising motif: 14 (3.3\%)

Expected frequency non-epitope: 0.2

Enrichment from expected: $\quad 69.9$

Chi-Square:

\begin{tabular}{|c|c|c|c|c|c|}
\hline Patient & TRBV & Deduced CDR3 $\beta$ sequence & TRBJ & Freq & Hyb ID \\
\hline 1041 & 27 & CASSLKGGAAHYF & $2-5$ & $5 / 103$ & $1041-c 7$ \\
\hline 1435 & 27 & CASSLKGGTGELFF & $2-2$ & $1 / 99$ & $1435-c 5$ \\
\hline 3421 & 27 & CASRLKGGSAEAFF & $1-1$ & $2 / 105$ & $3421-c 4$ \\
\hline \multirow[t]{2}{*}{1234} & $5-5$ & CASSLKGGGNQPQHF & $1-5$ & $3 / 93$ & $1234-c 7$ \\
\hline & $7-2$ & CASSLKAGGPGEQYF & $2-7$ & $2 / 93$ & $\mathrm{nt}^{1}$ \\
\hline \multirow[t]{3}{*}{8133} & 27 & CASS LKGGGPEAFF & $1-1$ & $1 / 94$ & $8133-c 4$ \\
\hline & 27 & CASS LKGGGEQFF & $2-1$ & $1 / 94$ & nt \\
\hline & 27 & CASS LKGGSYEQYF & $2-7$ & $1 / 94$ & $8133-c 4 r$ \\
\hline \multirow[t]{7}{*}{8845} & 27 & CASS LKGGGNGYTF & $1-2$ & $4 / 80$ & $8845-c 3$ \\
\hline & 27 & CASSLKGGGELFF & $2-2$ & $4 / 80$ & $8845-c 3 r$ \\
\hline & $7-2$ & CASSLKLAGG।VVTGELFF & $2-2$ & $6 / 80$ & $8845-c 1$ \\
\hline & $7-2$ & CASSLKASGGVHAETGTQYF & $2-5$ & $1 / 80$ & nt \\
\hline & $7-3$ & CASSLKASGG I S I NEQFF & $2-1$ & $1 / 80$ & $\mathrm{nt}$ \\
\hline & $7-3$ & CASS LKAGGLNTGELFF & $2-2$ & $1 / 80$ & $\mathrm{nt}$ \\
\hline & $7-3$ & CASS LKGAGGLGSTDTQYF & $2-3$ & $1 / 80$ & nt \\
\hline
\end{tabular}

${ }^{1}$ Hybridoma not made.

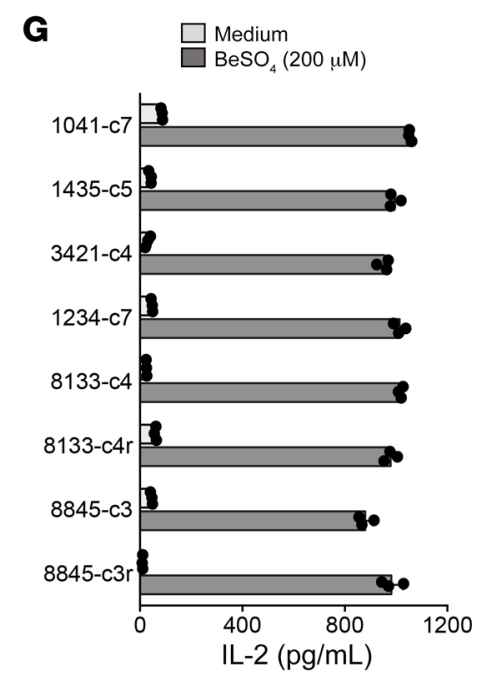

Figure 1. Identification of Be-specific BAL-derived T cells that express a CBD-related CDR3 $\beta$ motif. (A) Stacked bar representation of clonality found by single-cell

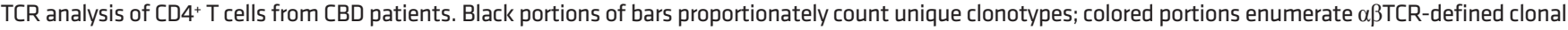
expansions with height proportional to the number of cells in the expansion. Total $\alpha \beta T C R$ pairs obtained for each patient are indicated. (B) Inverse Simpson's diversity plot of clone-size distributions. Displayed are means with upper and lower limits. (C) Cord diagram of T cell TR gene usage from CBD patients. Each individual T cell's TR gene usage profile is connected by a curved line whose thickness is proportional to the number of T cells with the respective gene pairing. Cenes are color coded based on frequency of usage, and enrichment of some genes relative to a background naive repertoire is indicated by arrows. (D) Two-dimensional PCA of TCRdist values for $\alpha \beta$ TCRs from all patients combined. Each dot represents a T cell, and color indicates usage of different TR genes. (E) TCR logo of CDR3 $\beta$ amino acid sequence motif identified in BAL-derived CBD T cells. Statistical significance was determined by $\chi^{2}$ analysis comparing the observed frequency of this motif versus the expected frequency. Panels show by proportion TRBV (left) and TRBJ (right) gene usage and encoded amino acid sequences (middle, top), respectively. Colored bars in the middle indicate the inferred nucleotide source for the corresponding position (black, TRD gene; red, $\mathrm{N}$-insertions). Bottom panel summarizes the likelihood of each amino acid residue being non-germline derived (taller is more likely). (F) TRB gene usage and junctional region amino acid sequence of T cells comprising the LKGGG CDR3 $\beta$ motif cluster. Red (and blue [K]) colors indicate nongermline or TRBD gene-encoded amino acids. Also shown are the number of identical TCRs over the total number of sequences obtained for each patient and TCRs selected to express in hybridomas. (G) IL-2 response of T cell hybridomas to medium and $\mathrm{BeSO}_{4}$ presented by splenic B cells isolated from HLA-DP2 transgenic mice. Data are representative of 3 experiments done in triplicate. 
sized dual-defined mixtures composed of varying combinations of aspartic acid/glutamic acid fixed at these 4 peptide positions. IL-2 secretion with these less diverse mixtures was only observed when there was a single aspartic acid and a single glutamic acid separated by 2 interceding amino acids (i.e., D4E7 or D5E8; Figure 2B). These data were confirmed by triple-defined mixtures that included phenylalanines as P1 and/or P6 anchor residues for HLA-DP2 binding (Figure 2C). Thus, mixtures composed of combinations of F1D4F6E7 or F2D5F7E8 were highly stimulatory at modest concentrations $(20 \mu \mathrm{g} / \mathrm{mL})$ and suggested that peptides binding in different registers were responsible for the doublets arising in the original unbiased library screening.

Based on these data, we generated a D5E8 biased library (D5E8 PSL) to perform a positional scan of the remaining 8 peptide positions (160 mixtures) in the context of fixed amino acids at p5 (aspartic acid) and p8 (glutamic acid). In addition, our investigation was expanded to include 4 of the LKGGG CDR3 $\beta$ TCRs (8133-c4r, 8845-c3, 8133-c4, 8845-c3r) tested at multiple concentrations of peptide mixtures. Representative results of 2 hybridomas screened with the mixtures $(20 \mu \mathrm{g} / \mathrm{mL})$ and $\mathrm{BeSO}_{4}(75 \mu \mathrm{M})$ are shown in Figure 2D, and normalized results for the 4 hybridomas in response to the library at $50 \mu \mathrm{g} / \mathrm{mL}$ are depicted in Supplemental Figure 4. Of note, a hybridoma (DV-13) expressing an HLA-DP2-restricted TCR specific to a dengue virus NS3 peptide (254-265; REIVDLMCHATF) was not stimulated by the D5E8 PSL (Figure 2E; p2D5E8 and data not shown). While demonstrating individual preferences, these 4 TCRs importantly also shared distinct amino acid profiles at particular positions, as predicted based on their close TCRdist measurements. For example, preferences for phenylalanine followed by cysteine and other hydrophobic amino acids were observed at the $\mathrm{p} 2$ and $\mathrm{p} 7$ positions, indicative of favored anchor residues for HLA-DP2 at the P1 and P6 positions within the binding groove $(10,16,17)$. A prominent common preference was a tyrosine at the $\mathrm{p} 6$ position of the peptide. Because of its position within the HLA-DP2-binding groove (P5), this residue is likely facing up and making multiple contacts with each interacting TCR. A sequence logo quantitatively portraying amino acid preferences at all peptide positions for each of the hybridomas is displayed in Figure 3A.

To determine whether Be-dependent mimotopes that stimulate all 4 TCRs could be ascertained from the biased library screen, we synthesized 32 peptides that were selected by choosing amino acids at each position of the D5E8 PSL that generated the most active hybridoma responses. These were tested in the presence and absence of $\mathrm{BeSO}_{4}$ for hybridoma IL-2 responses (amino acids chosen at each position indicated in Figure 2D and sequences listed in Supplemental Figure 5A). As shown in Supplemental Figure $5 \mathrm{~A}$, hybridomas $8133-\mathrm{c} 4 \mathrm{r}$ and 8845 -c $3 \mathrm{r}$ recognized all mimotopes, 8845-c3 recognized 28, and 8133-c4 was the most restricted in its recognition, showing activity to 4 of the 32 peptides. A hybridoma (AV22) expressing a lung-derived TCR specific for the HLA-DP2plexin A/Be complex (11) did not respond to any peptides at $5 \mu \mathrm{g} /$ $\mathrm{mL}$ (data not shown). In contrast to the other more promiscuous TCRs, the TCR expressed on hybridoma 8133-c4 required a nonpolar aliphatic amino acid at p4 (isoleucine), a bulky hydrophobic phenylalanine rather than leucine at $\mathrm{p} 7$, and responded to peptides with a serine at $\mathrm{p} 9$ rather than tryptophan. Dose-response curves were completed for the 4 mimotopes that stimulated all of the hybridomas, and representative results are shown for 8133-c4 (Supplemental Figure 5B). These mimotopes differed only at $\mathrm{p} 1$ (valine or tryptophan) and p3 (arginine or valine), and $\mathrm{EC}_{50}$ values (peptide concentration inducing half-maximal IL-2 response) fell within a relatively narrow range $(17-151 \mathrm{ng} / \mathrm{mL}$, Supplemental Figure $5 \mathrm{C}$ ). These data confirmed that Be-dependent mimotopes with crossreactivity to the related set of LKGGG CDR3 $\beta$ TCRs could readily be identified.

Naturally occurring peptides recognized by the LKGGG CDR $3 \beta$ $T$ cell receptors. We utilized biometrical analysis to identify candidate naturally occurring human peptides capable of stimulating the Be-specific LKGGG CDR3 $\beta$ T cell hybridomas. Scoring matrices were created based on the stimulatory potential of each amino acid at each position of the D5E8 PSL. A total of 18 matrices were created, and these matrices were applied to all possible overlapping decapeptides in the UniProt human protein database to rank peptides based on the sum of the matrix values for each amino acid in the peptide. Since all 4 hybridomas were screened against the biased D5E8 PSL, with some at several different concentrations, multiple independent biometrical analyses were performed. The resulting lists of potential antigens were consolidated by taking the top 100 peptides by matrix score for each data set and merging the lists based on each sequence's best rank in any analysis. Thus, this selection process prioritized peptides ranked highly in at least one analysis over peptides present on multiple lists, but having mediocre ranking. In total, any peptide ranked in the top 25 of any analysis was considered, provided it comprised both D5 and E8 amino acids and had no more than one cysteine, so as to conform to the makeup of the library tested.

The number of $\mathrm{T}$ cell hybridomas used to assess activity of the natural peptides was expanded to 8 to incorporate representatives of the LKGGG CDR3 $\beta$ motif that were independent of the biometrical analyses and to include TCRs from each of the CBD patients enrolled in the study. Supplemental Table 2 orders the 100 peptides synthesized by score, listing their protein source, ranking in selected subanalyses, and IL-2 secretion by the $8 \mathrm{~T}$ cell hybridomas in response to peptide in the presence of $\mathrm{BeSO}_{4}$. In total, 19 peptides stimulated ( $>200 \mathrm{pg} / \mathrm{mL} \mathrm{IL-2}$ ) at least 1 of the hybridomas at $1 \mu \mathrm{g} / \mathrm{ml}$ (Figure $3 \mathrm{~B}$ ). The number of peptides that activated the hybridomas ranged from only 3 by hybridoma $8845-c 3 r$ to 11 by hybridomas 8133-c4r and 3421-c4. Remarkably, the highest ranked peptide from the biometrical analyses (BA001) stimulated all of the $\mathrm{T}$ cell hybridomas tested, and all maintained high levels of activity at the lower concentration of peptide $(0.2 \mu \mathrm{g} / \mathrm{mL})$ (Figure 3B). This peptide was derived from the CCL4 protein (CCL4 $4_{23-32}$ ). The second ranked peptide (BA002) was also derived from a CCL3 protein $\left(\mathrm{CCL} 3_{22-31}\right.$ ) and activated 7 of 8 hybridomas (excluding 8845-c3r). Other peptides derived from C-C motif chemokines were also stimulatory to the majority of hybridomas (BA018 and BA021; Figure 3B). Figure 3C highlights the amino acid similarities between the recognized CCL4 and CCL3 epitopes. Thus, most of the TCRs were crossreactive to 2 highly similar peptides derived from chemokines involved in the innate immune response.

Fine specificity of response to CCL peptides by LKGGG CDR $3 \beta$ hybridomas. To investigate different aspects of recognition of the CCL/Be ligands, we performed an alanine scan of the CCL4 
A

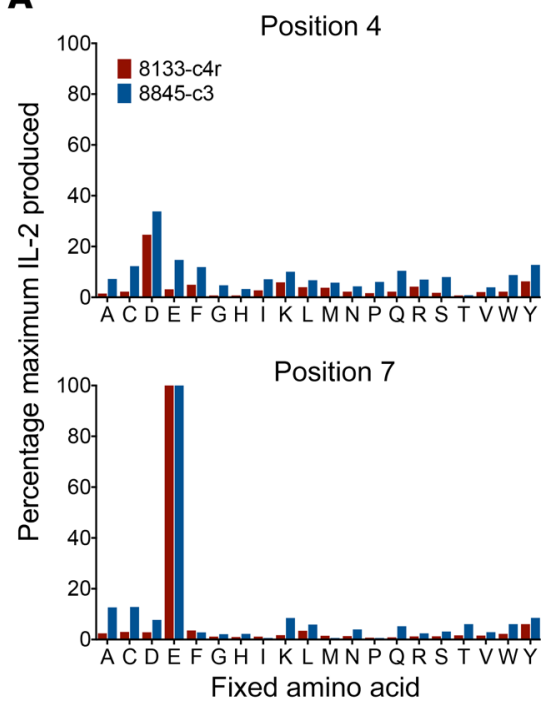

D
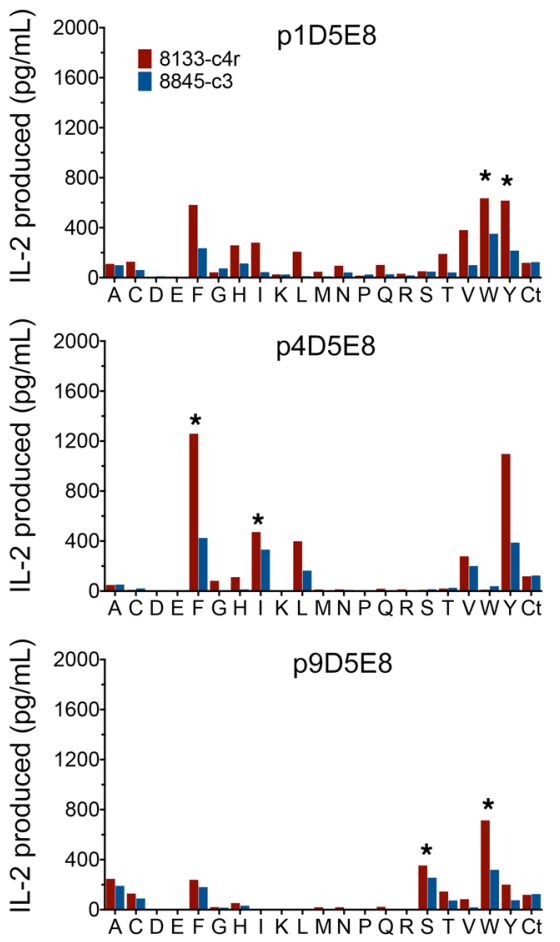

Fixed amino acid
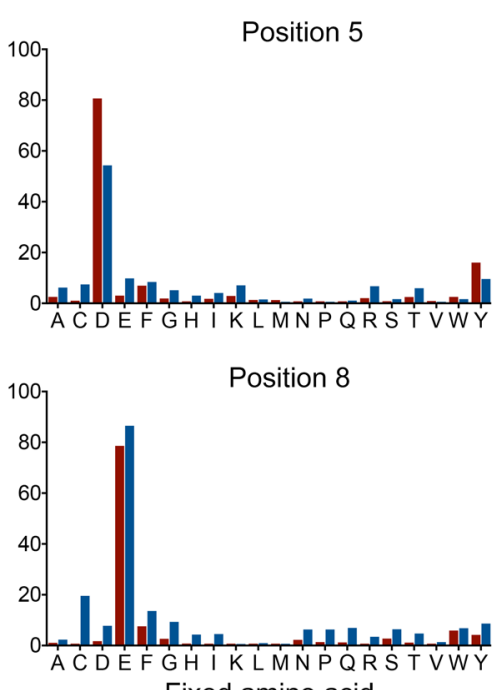

Fixed amino acid
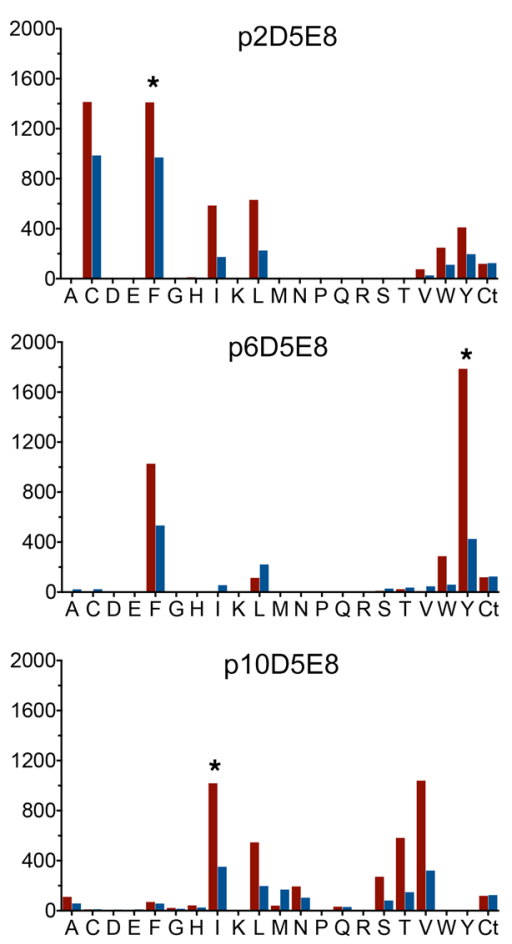

Fixed amino acid
B

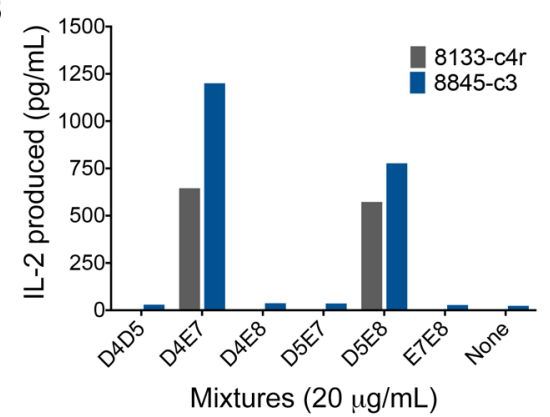

\section{C}
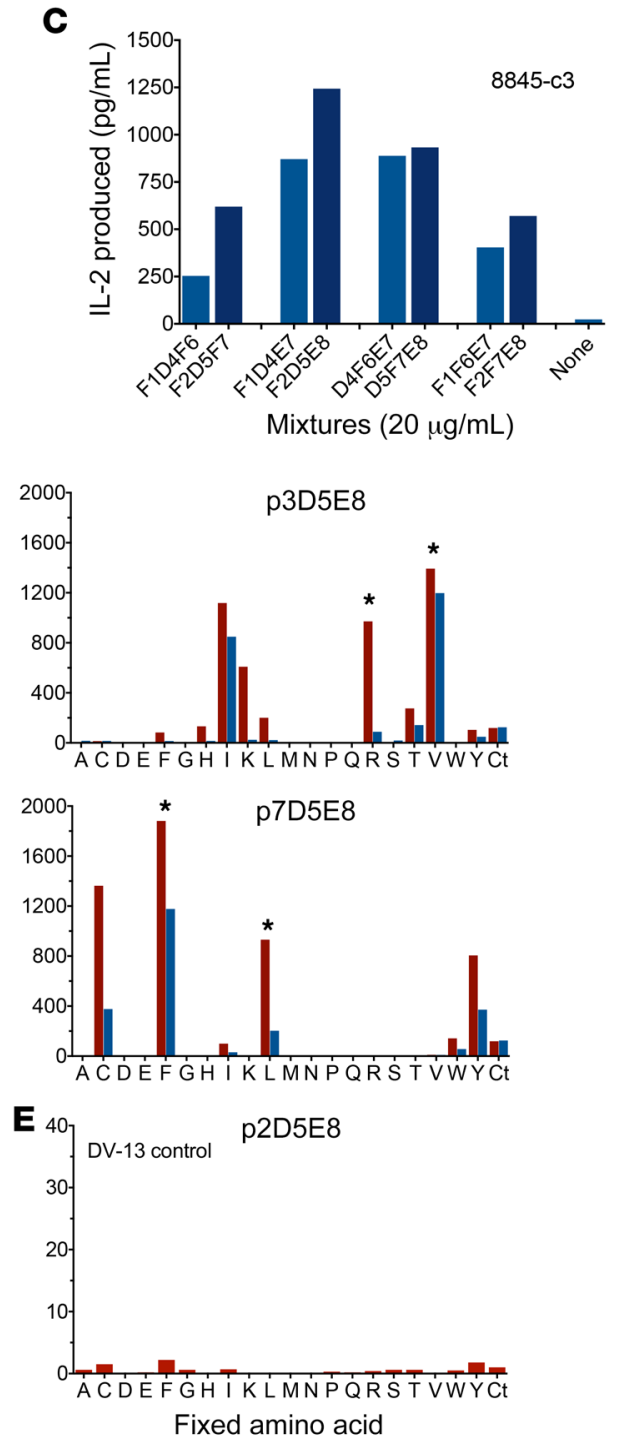

Figure 2. Antigen discovery of BAL-derived LKGGG CDR3 $\beta$ TCRs using unbiased and biased decapeptide PSLs. (A) Representative results of 2 experiments depicting IL-2 responses (pg/mL, done in duplicate) of hybridomas $8133-c 4 \mathrm{r}$ and $8845-\mathrm{c} 3$ to an unbiased PSL. Peptide mixtures $(200 \mu \mathrm{g} / \mathrm{mL}$ ) were presented by HLA-DP2-transfected fibroblast line DP2.21 in the presence of $\mathrm{BeSO}_{4}(75 \mu \mathrm{M})$, and data were normalized to peak IL-2 produced in the assay for each hybridoma (mixture E7, lower left). Label on $x$ axis denotes amino acid (single letter code) fixed at each defined position. Four (p4, p5, p7, p8) of 10 peptide positions scanned are displayed. (B and C) IL-2 produced $(\mathrm{pg} / \mathrm{mL})$ by indicated hybridomas in response to select dual-defined $(100 \mu \mathrm{g} / \mathrm{mL})(\mathbf{B})$ and triple-defined $(20 \mu \mathrm{g} / \mathrm{mL})(\mathbf{C})$ mixtures in the presence of $\mathrm{BeSO}_{4}(75 \mu \mathrm{M})$. (D and $\left.\mathbf{E}\right) \mathrm{IL}-2$ responses $(\mathrm{pg} / \mathrm{mL})$ of hybridomas $8133-\mathrm{c} 4 \mathrm{r}$ and $8845-\mathrm{c3}(\mathbf{D})$ and hybridoma DV-13 (E) to a biased PSL with 2 positions fixed (D5E8). Each panel shows a different peptide position scanned using peptide mixtures (20 $\mu \mathrm{g} / \mathrm{mL}$ ) tested in duplicate in the presence of $\mathrm{BeSO}_{4}$ and including a control D5E8 mixture (Ct). Asterisks indicate amino acids selected at each peptide position for synthesis of mimotopes. 
A
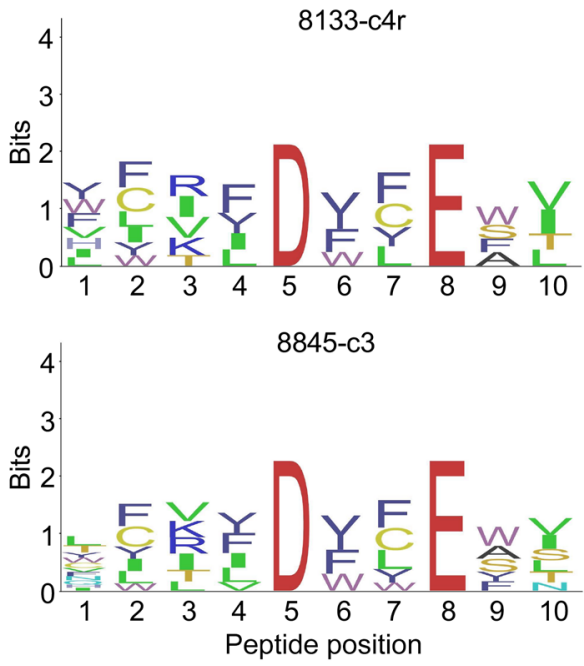

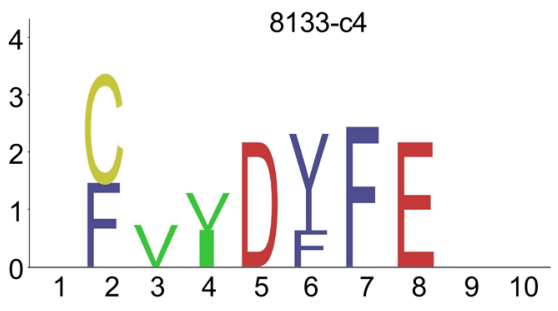

$8845-c 3 r$

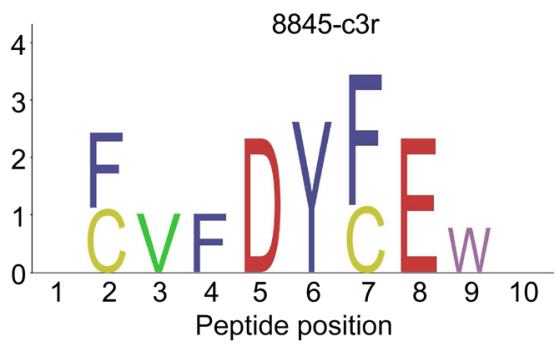

\begin{tabular}{|c|c|c|c|c|c|c|c|c|c|c|c|c|c|c|c|c|c|c|c|}
\hline \multirow{3}{*}{ ID } & \multirow{3}{*}{ Peptide } & \multicolumn{16}{|c|}{$\mathrm{IL}-2$ response $(\mathrm{pg} / \mathrm{mL})$ of hybridomas at 1.0 and $0.2 \mu \mathrm{g} / \mathrm{mL}$ peptide } & \multirow{3}{*}{$\begin{array}{c}\text { No. hybs } \\
>200 \mathrm{pg} / \mathrm{mL} \\
\mathrm{IL}-2 \text { at } \\
1 \mu \mathrm{g} / \mathrm{mL}\end{array}$} & \multirow{3}{*}{ Human Uniprot ID and protein name } \\
\hline & & \multicolumn{2}{|c|}{$8845-c 3$} & \multicolumn{2}{|c|}{$8845-c 3 r$} & \multicolumn{2}{|c|}{$8133-c 4$} & \multicolumn{2}{|c|}{$8133-c 4 r$} & \multicolumn{2}{|c|}{$1041-c 7$} & \multicolumn{2}{|c|}{$1435-c 5$} & \multicolumn{2}{|c|}{$3421-c 4$} & \multicolumn{2}{|c|}{$1234-\mathrm{c} 7$} & & \\
\hline & & 1 & 0.2 & 1 & 0.2 & 1 & 0.2 & 1 & 0.2 & 1 & 0.2 & 1 & 0.2 & 1 & 0.2 & 1 & 0.2 & & \\
\hline BA001 & NFVVDYYETS & 388 & 415 & 420 & 294 & 426 & 319 & 365 & 420 & 717 & 470 & 694 & 461 & 651 & 496 & 602 & 453 & 8 & Q8NHW4|CC4L C-C motif chemokine 4-like \\
\hline BA002 & NFIADYFETS & 431 & 492 & - & - & 422 & 481 & 393 & 499 & $730 !$ & 536 & 512 & 136 & 665 & 529 & 608 & 502 & 7 & P10147|CCL3 C-C motif chemokine 3 \\
\hline BA003 & FFRYDFFERI & - & 4.1 & - & - & - & - & 283 & 310 & - & - & 12 & - & 413 & 97 & - & - & 2 & A0A126LAV1|U7 \\
\hline BA005 & YFRVDFYEAM & 434 & 556 & 428 & 472 & - & - & 379 & 553 & 702 & 585 & 733 & 588 & 687 & 599 & - & - & 6 & $\begin{array}{l}\text { Q510X7|TTC32 Tetratricopeptide repeat } \\
\text { protein } 3\end{array}$ \\
\hline BA008 & QLVVDWLESI & 451 & 285 & 302 & 55 & - & - & 213 & 20 & 621 & 188 & 591 & 150 & 271 & 21 & - & - & 6 & $\begin{array}{l}\text { P57740|NU107 Nuclear pore complex } \\
\text { protein Nup107 }\end{array}$ \\
\hline BA009 & HFILDFYEKV & 400 & 308 & - & - & - & - & 7.2 & - & - & - & - & - & - & - & - & - & 1 & Q92674|CENPI Centromere protein I \\
\hline BA011 & KFVDDLFETV & 7 & 1.4 & - & - & - & - & 1.3 & - & 1.1 & - & - & - & 330 & 11 & - & - & 1 & P51805|PLXA3 Plexin-A3 \\
\hline BA014 & DFIYDLFEHV & 424 & 498 & - & - & - & - & 388 & 429 & 5.1 & - & 488 & 114 & 429 & 92 & - & - & 4 & Q9HD67|MYO10 Unconventional myosin-X \\
\hline BA015 & RFTTDYFEVS & 5.5 & - & - & - & - & - & 4.2 & - & 2.4 & - & 2.7 & - & 620 & 247 & - & - & 1 & Q96PH6|DB118 Beta-defensin 118 \\
\hline BA018 & KFIVDYSETS & 476 & 18 & - & - & 197 & - & 271 & - & 590 & 11 & 278 & - & 541 & 22 & 549 & 12 & 6 & P55774|CCL18 C-C motif chemokine 18 \\
\hline BA021 & NFMADYFETS & 115 & 3.8 & - & - & 769 & 485 & 642 & 297 & 702 & 504 & - & - & 617 & 175 & 663 & 185 & 5 & $\begin{array}{l}\text { Q14745|Q14745 C-C motif chemokine } \\
\text { (Fragment) }\end{array}$ \\
\hline BA041 & KIIADIFEYT & 4.6 & - & - & - & 666 & 55 & - & - & - & - & 4 & - & 1.8 & - & - & - & 1 & $\begin{array}{l}\text { P22033|MUTA Methylmalonyl-CoA mutase, } \\
\text { mitochondrial }\end{array}$ \\
\hline BA048 & NYIYDLLEEV & 406 & 145 & - & - & - & - & 86 & - & - & - & 11 & - & 25 & - & - & - & 1 & Q02241|KIF23 Kinesin-like protein KIF23 \\
\hline BA053 & ELIFDFFEED & 34 & 7.1 & - & - & - & - & 142 & 0.9 & 603 & 108 & - & - & - & - & - & - & 1 & $\begin{array}{l}\text { Q9NPB8|GPCP1 Glycerophosphocholine } \\
\text { phosphodiesterase GPCPD1 }\end{array}$ \\
\hline BA060 & FLVFDLWEDT & 432 & 540 & - & - & - & - & 398 & 477 & 73 & - & 673 & 572 & - & - & - & - & 3 & $\begin{array}{l}\text { Q16816-2|PHKG1 Isoform } 2 \text { of } \\
\text { Phosphorylase b kinase gamma catalytic } \\
\text { chain, skeletal muscle/heart isoform }\end{array}$ \\
\hline BA065 & FLLTDYFEED & 7.8 & - & - & - & 28 & - & 17 & - & 478 & 4.4 & 3.7 & - & - & - & 683 & 71 & 2 & Q8IVI9|NOSTN Nostrin \\
\hline BA076 & PFSFDFFEDP & 707 & 194 & - & - & - & - & 382 & 0.6 & 31 & - & - & - & 359 & 8 & - & - & 3 & $\begin{array}{l}\text { O75190|DNJB6 DnaJ homolog subfamily B } \\
\text { member } 6\end{array}$ \\
\hline BA081 & QLLVDFWEAQ & 74 & 3.5 & - & - & - & - & - & - & 383 & - & - & - & - & - & - & - & 1 & $\begin{array}{l}\text { Q969F9|HPS3 Hermansky-Pudlak } \\
\text { syndrome } 3 \text { protein }\end{array}$ \\
\hline BA084 & DYLFDFFEHL & 1.3 & 6.6 & - & - & - & - & 203 & 57 & 94 & - & - & - & - & 1.6 & - & - & 1 & $\begin{array}{l}\text { Q9NPP4|NLRC4 NLR family CARD domain- } \\
\text { containing protein } 4\end{array}$ \\
\hline
\end{tabular}

C

\begin{tabular}{ccc|cc|cc|c|ccc} 
Human peptide (Uniprot No.) & \multicolumn{5}{c}{ Amino acid sequence } \\
\hline CCL4(23-32) (P13236) & N & F & V & V & D & Y & Y & E & T & S \\
CCL3(22-31) (P10147) & N & F & I & A & D & Y & F & E & T & S \\
\hline
\end{tabular}

Figure 3. Identification of Be-dependent naturally occurring peptides that stimulate T cell hybridomas expressing the LKGGG CDR3 $\beta$ motif. (A) Sequence logos summarizing hybridoma responses to the D5E8 PSL are shown, depicting amino acids fixed at the listed peptide position by hybridoma activity. Only amino acids with greater than $25 \%$ of maximal activity for each hybridoma are shown, and the height of each amino acid (single-letter code) corresponds to the activity of the peptide. If no amino acids are displayed, none met the threshold. The color of each letter groups amino acids by their chemical characteristics. (B) Response of 8 hybridomas expressing LKGGG CDR3 $\beta$ TCRs to naturally occurring peptides plus BeSO ${ }_{4}$ presented by HLADP2-transfected fibroblasts. Peptides are ordered by their biometrical analysis ranking and are included on the list only if at least one of the hybridomas demonstrated a positive response ( $200 \mathrm{pg} / \mathrm{mL}$ IL-2 at $1.0 \mu \mathrm{g} / \mathrm{mL}$ peptide). Intensity of green color highlights peptides inducing the highest IL-2 secretion. The protein source of each peptide is indicated by its UniProt identification number and name. (C) UniProt number and amino acid sequence of related human chemokine peptides derived from CCL4 and CCL3 (biometrical analysis peptides BA001 and BA002, respectively). 
peptide and tested hybridomas from CBD patients 8845 and 8133 (Figure 4, A and B, and Supplemental Figure 6). Among the shared properties of these TCRs, substitutions at the p5, p6, and p8 positions of the peptide abrogated TCR recognition, as indicated by loss of IL-2 secretion, while substitutions at p1 and p10 had minimal effect. When the bulky hydrophobic residues at the anchoring positions (i.e., P1 and P6) of HLA-DP2 were substituted for alanines, all hybridomas displayed either a pronounced (F2A) or moderate (Y7A) diminution in IL-2 secretion in response to peptide. The most variable responses among the hybridomas occurred at positions p3 (valine), p4 (valine), and p9 (threonine). In contrast to the other hybridomas, the V3A substitution abolished IL-2 secretion by hybridoma 8845 -c3r. These data are consistent with this hybridoma not recognizing the CCL3 peptide that has an isoleucine at that position (Figure 3B).

To examine the $\mathrm{N}$ - and $\mathrm{C}$-terminus amino acids required for recognition of the CCL4 and CCL3 peptides, we completed dose-response curves to peptides of varying lengths (Figure 4, C and D, and Supplemental Tables 3 and 4). Based on the overall response patterns for the 4 hybridomas, the core epitope for both CCL4 and CCL3 peptides starts with phenylalanine, likely the P1 anchor residue for HLA-DP2 binding (Figure 4, C and D, and Supplemental Tables 3 and 4). Removal of this amino acid abrogated the response to CCL4 (Figure 4C and Supplemental Table 3). Furthermore, addition of likely $\mathrm{P}-1$ ( $\mathrm{N}$ for both peptides) and $\mathrm{P}-2$ ( $\mathrm{R}$ for CCL4) HLA-DP2 binding residues had negligible impact on peptide recognition, and hybridoma responses were uniformly better when phenylalanine was the first amino acid in the peptide. Thus, a 10-mer starting with phenylalanine for both peptides appears to be the maximal epitope for these 4 hybridomas. Removal of the putative $\mathrm{P} 9$ and $\mathrm{P} 10$ binding residues (serines in both peptides) to create CCL4 and CCL3 8-mer peptides (starting with phenylalanine) still retained activity and demonstrated that these 2 amino acids have a modest effect on $\mathrm{EC}_{50}$ values (Figure $4, \mathrm{C}$ and $\mathrm{D}$, and Supplemental Tables 3 and 4). Finally, removal of glutamic acid (6-mer peptide starting with phenylalanine) abrogated $\mathrm{T}$ cell recognition (Figure 4C and Supplemental Table 3), suggesting that the CCL4 minimal epitope for Be-induced $\mathrm{T}$ cell activation is a 7-mer consisting of FVVDYYE, with the $\mathrm{F}$ representing the well-defined P1 anchor residue for HLA-DP2 binding $(10,16,17)$.

Results from the unbiased PSL screens of hybridomas 8133c4r and 8845-c3 showed a clear preference for the combination of D5E8 (P4 and P7 for HLA-DP2 binding) due to spacing considerations for tetrahedral coordination of the $\mathrm{Be} 2^{+}$moiety within the HLA-DP2-binding groove (10). To determine how readily TCRs could tolerate different acidic amino acids at those Be-coordinating positions, we synthesized variants of the CCL4 peptide, varying positions p5 and p8 (Figure 4E). Interestingly, 3 of 4 hybridomas allowed a glutamic acid at position 5 (E5), although the $\mathrm{EC}_{50}$ values for this change were 3- to 40-fold higher than the native peptide (Figure 4E). Hybridoma 8133-c4 was also activated by the D5D8 and E5D8 variants, having $\mathrm{EC}_{50}$ values ranging from 58 to $206 \mathrm{nM}$.

TCR crossreactivity between CCL and plexin peptides. Since we had determined numerous $\alpha \beta T C R$ pairs expressed in the lungs of CBD patients, we questioned whether any non-LKGGG CDR3 $\beta$ TCRs recognized the CCL peptides. Hybridomas were generated expressing the 4 additional TCRs shown in Supplemental Figure
7A, representing either a BAL-derived clonal expansion (8845-c2) or T cells expressing CDR3 $\beta$ motifs related to other TCRs (1435c1r, 3421-c2r). We also created another LKGGG-based hybridoma (8845-c1) that, while part of the motif based on TCRdist (Figure $1 F)$, had an extended CDR3 $\beta$ amino acid sequence that looked quite different from the other TCRs in the set. The non-LKGGG hybridomas were unresponsive to a subset of naturally occurring human peptides tested at high concentration $(5 \mu \mathrm{g} / \mathrm{mL}$, Supplemental Figure 7B) in the presence of Be. However, hybridoma 8845-c1 responded to both chemokine peptides as well as to a previously identified Be-dependent naturally occurring peptide, plexin A4 (11), ranked number 7 on our list. In the initial screening of the biometrical analysis peptides, hybridoma 3421-c4 also recognized a plexin peptide (plexin A3, BA011; Figure 3B).

To dissect the crossreactive responses observed with hybridoma 8845-c1, we examined a set of $\mathrm{p} 4$ and $\mathrm{p} 6$ variant peptides that bridged the primary differences between the CCL4 and plexin A4 epitopes (Figure 5A). Hybridoma 8845-c1 responded to all peptides (Figure 5B), although the $\mathrm{EC}_{50}$ value for CCL4 was significantly lower than that for the plexin A4 peptide (data not shown). In contrast, hybridoma AV22 showed no crossreactivity to the CCL4 peptide (Figure 5B). The AV22 TCR tolerated a change from aspartic acid at position 4 to valine, but changing the leucine at position 6 to tyrosine blocked recognition. This observation was corroborated by the positive recognition of the CCL4-6L peptide. The other 8 LKGGG CDR3 $\beta$ hybridomas were tested against this set of peptides (Supplemental Figure 8), and none displayed crossreactivity to plexin; most (excepting 8845 -c3r) partially responded only to the plexin A-4V6Y peptide, indicating a requirement for both peptide positions 4 and 6 to resemble the CCL4 epitope.

Validation of chemokine-derived peptides as Be-dependent antigens in CBD. As a first step to prove chemokine/Be neoantigens are targeted by the adaptive immune response in CBD patients, we ascertained whether chemokine proteins can be processed to yield the stimulatory HLA-DP2 binding epitopes. Recombinant purified CCL4 and CCL3 proteins were tested at varying concentrations using HLA-DP2-transfected fibroblasts as antigen-presenting cells. IL-2 secretion was observed from hybridomas 8133-c4r and 8845c3 at low concentrations of protein and only in the presence of Be (Figure 6A). As expected, hybridoma 8845-c3r did not respond to the CCL3 protein (Figure 6A), and neither protein stimulated the HLA-DP2-restricted DV-13 hybridoma expressing a TCR specific to a dengue virus NS3-derived epitope (data not shown).

An important consideration for validating antigens is to demonstrate that they are present or can be induced at the site of disease activity. Thus, we determined levels of CCL4 and CCL3 chemokines in BAL cells from CBD patients cultured in vitro in either medium or stimulated with $\mathrm{BeSO}_{4}$ (Figure 6B). While some patients had undetectable chemokines after culture in medium, most exhibited wide-ranging values of media-induced chemokines (up to $45,000 \mathrm{pg} / \mathrm{mL}$ CCL4 and 85,000 pg/mL CCL3). Addition of $\mathrm{BeSO}_{4}$ to the cell cultures induced a large release of both chemokines, particularly in those patients with lower baseline levels of these proteins (Figure 6B).

Next, HLA-DP2 tetramers were constructed with CCL4 and CCL3 peptides covalently attached to the N-terminus of the HLADP2 $\beta$-chain and saturated in $\mathrm{BeSO}_{4}$ (11). These reagents only 
A
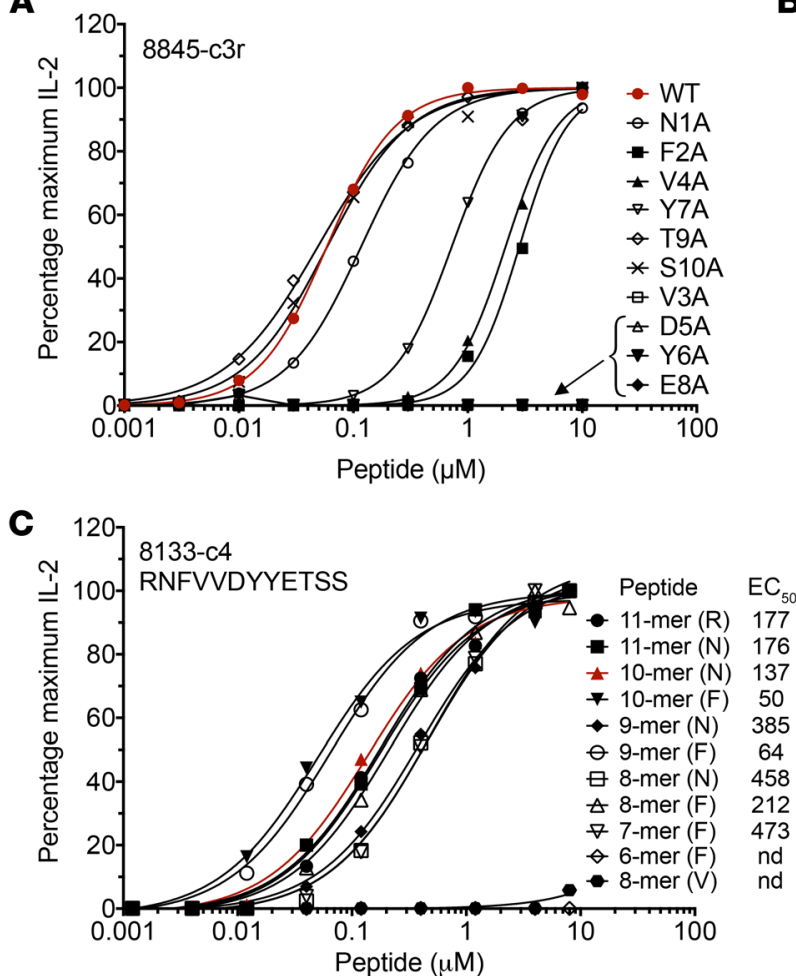

$\mathbf{E}$

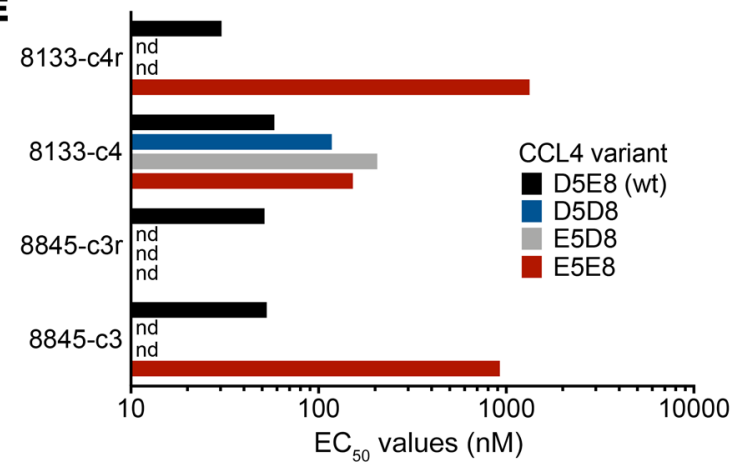

B

\begin{tabular}{|c|c|c|c|c|c|c|}
\hline \multicolumn{3}{|c|}{ Peptide } & \multicolumn{4}{|c|}{$\mathrm{EC}_{50}(\mathrm{nM})$ for Hybridoma } \\
\hline 1 & $\begin{array}{lllllll}2 & 3 & 4 & 5 & 6 & 7 & 8 \\
\end{array}$ & 910 & $8845-c 3$ & $8845-c 3 r$ & 8133-c4 & $8133-c 4 r$ \\
\hline $\mathrm{N}$ & $F \vee V D Y Y E$ & $T S$ & 89.1 & 58.8 & 58.1 & 24.7 \\
\hline A & $F \vee V D Y Y E$ & T S & 54.3 & 116.9 & 48.5 & 15.6 \\
\hline $\mathrm{N}$ & $A \vee V D Y Y E$ & $\mathrm{~T} S$ & 907.5 & 2822.0 & 1624.0 & 542.8 \\
\hline & $F A \vee D Y Y E$ & $\mathrm{~S}$ & 278.3 & $\mathrm{nd}^{*}$ & 236.2 & 614.2 \\
\hline $\mathrm{N}$ & $F \vee A D Y Y E$ & T S & 71.3 & 2139.0 & 202.8 & 31.9 \\
\hline $\mathrm{N}$ & $F \vee V A Y Y E$ & $\mathrm{~T} \mathrm{~S}$ & nd & nd & nd & nd \\
\hline $\mathrm{N}$ & $F \vee \vee D A Y E$ & $T S$ & nd & nd & nd & nd \\
\hline $\mathrm{N}$ & $F \vee V D Y A E$ & $\mathrm{~S}$ & 443.8 & 724.5 & 730.5 & 257.0 \\
\hline $\mathrm{N}$ & $F \vee V D Y Y A$ & $\mathrm{~T} S$ & nd & nd & nd & nd \\
\hline & $F \vee V D Y Y E$ & A S & 241.7 & 49.0 & 1734.0 & 43.3 \\
\hline & $F \vee \vee D Y Y E$ & $\mathrm{~T} A$ & 58.9 & 59.2 & 42.8 & 23.2 \\
\hline
\end{tabular}

*nd, Not determined under conditions studied.

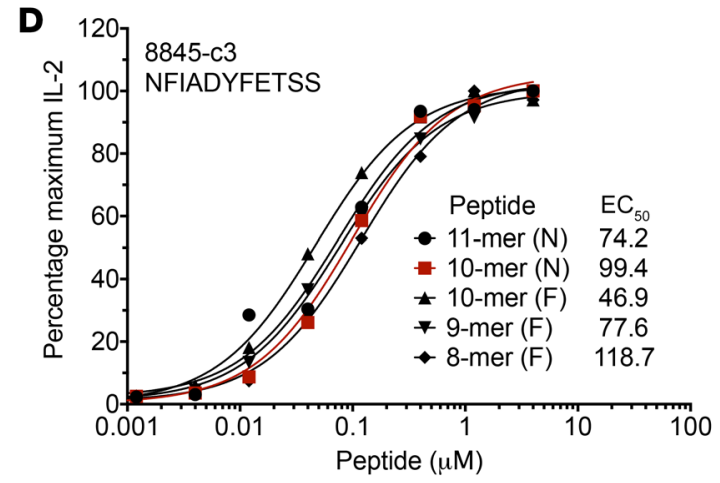

Figure 4. Characterization of human chemokine-derived peptides that stimulate Be-specific $T$ cell hybridomas expressing the LKGGC CDR3 $\beta$ CBD public motif. (A) Dose-response curves to CCL4 peptides with single alanine substitutions are shown for representative hybridoma 8845-c3r. Data are plotted as the percentage of maximum IL-2 secretion against peptide concentration in the presence of $\mathrm{BeSO}_{4}$. The natural CCL4 peptide (WT) curve is drawn in red. (B) Summary of CCL4 peptide alanine scan for $4 \mathrm{~T}$ cell hybridomas. $\mathrm{EC}_{50}$ values $(\mathrm{nM})$ are presented for a representative experiment and are color coded based on peptide activity (green, high; yellow, moderate; orange, negative). (C and D) Dose-response curves of representative hybridomas 8133-c4 (C) and 8845-C3 (D) in response to length variants of the CCL4 (C) and CCL3 (D) peptides are shown. Length and first amino acid (single-letter code) of each peptide is indicated, and $\mathrm{EC}_{50}$ values (nM) of each experiment are displayed. (E) EC $\mathrm{C}_{50}$ values (nM) quantitating T cell hybridoma ( $\left.n=4\right)$ responses to CCL4 peptides with varying acidic amino acid composition (aspartic acid or glutamic acid) at positions $\mathrm{p} 5$ and $\mathrm{p} 8$ are shown. Data are representative of 2 separate experiments. nd, not determined due to low responses.

stained hybridomas expressing TCRs specific for the CCL3/CCL4 epitopes (representative results, Figure 6C). Neither AV22 nor the dengue virus-specific hybridoma DV-13 bound these Be-pulsed HLA-DP2-CCL tetramers. Hybridoma 8845-c3r only bound the CCL4-based tetramer, while 8845-c1 stained both chemokine tetramers and a DP2-plexin A/Be tetramer, reflecting its crossreactivity to these epitopes. Tetramer binding for each of the TCRs matched the degree of $\mathrm{T}$ cell hybridoma activation as determined by IL-2 production.

$\mathrm{HLA}^{-D P 2}{ }^{+}$and -DP2- CBD patients as well as Be-sensitized (BeS) subjects were selected to investigate the frequency of HLA-
DP2-CCL4/Be- and -CCL3/Be-specific CD4 ${ }^{+}$T cells among ex vivo BAL cells. BeS subjects had a history of Be exposure, but had normal lung function and lacked a Be-specific adaptive immune response in the lung. Thus, this cohort served as a disease control to confirm the specificity of the tetramers. For the 10 HLA-DP2 ${ }^{+}$CBD subjects, the frequency of tetramer-binding $\mathrm{CD} 4^{+} \mathrm{T}$ cells ranged broadly from less than $0.1 \%-22.0 \%$ for HLA-DP2-CCL4/Be $(3.9 \% \pm 2.2 \%$, mean \pm SEM), and less than 0.1\%-25.0\% for the HLA-DP2-CCL3/ Be tetramer $(4.1 \% \pm 2.5 \%$; Figure $6 \mathrm{D})$. As shown in Figure $6 \mathrm{D}$, minimal or no HLA-DP2-chemokine/Be tetramer-binding CD4 ${ }^{+}$T cells were observed in the BAL of HLA-DP2 ${ }^{+}$BeS subjects. 
A

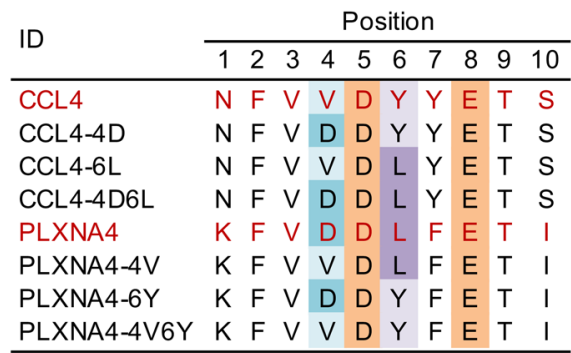
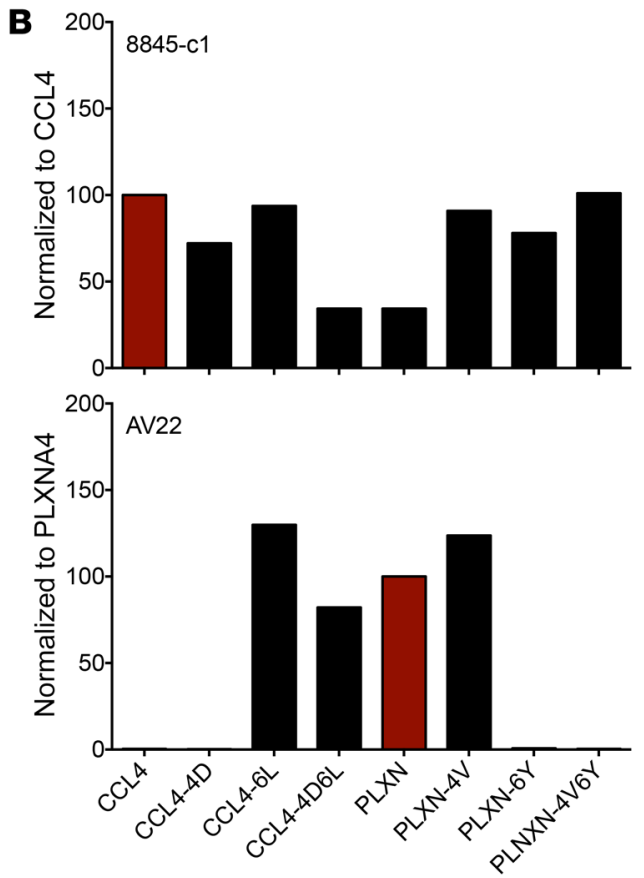

Figure 5. An LKGGC CDR3 $\beta$ TCR crossreactive to CCL4 and PLXNA4 peptides. (A) Set of CCL4 and PLXNA4 WT (red) and variant peptides that differed at the $p 4$ and $p 6$ positions. Colors highlight D5E8 and amino acids that vary at $\mathrm{p} 4$ and p6. (B) IL-2 production by hybridomas 8845-c1 (top) and AV22 (bottom) in response to CCL4 and PLXNA4 WT and variant peptides presented by HLA-DP2-transfected fibroblasts at $300 \mathrm{ng} / \mathrm{mL}$ in the presence of $\mathrm{BeSO}_{4}$. Data were normalized to WT peptides (CCL4 for 8845-c1; PLXNA4 for AV22) and are representative of 2 experiments done in duplicate.

Tetramer staining was coupled with intracellular cytokine staining (ICS) for Be-induced IFN- $\gamma$, the Th1-type cytokine most abundantly expressed in the $\mathrm{CBD}$ lung and a marker for $\mathrm{CD} 4^{+} \mathrm{T}$ cell activation (9). Thus, this assay enabled investigation of the number of chemokine/Be-specific cells in relation to the overall number of Be-specific $\mathrm{CD} 4^{+} \mathrm{T}$ cells in the $\mathrm{BAL}$, and these data are summarized for the HLA-DP2 ${ }^{+} \mathrm{CBD}$ cohort in Figure 6E. Despite the broad range of Be-induced IFN- $\gamma$-secreting cells among the patients with active disease (range, 0.15\%-33\%), consistently high fractions of chemokine/Be-specific cells were detected among these activated $\mathrm{T}$ cells (CCL4, $18.7 \% \pm 4.2 \%$; CCL3, $14.1 \%$ $\pm 3.8 \%)$. In contrast, no Be-induced IFN- $\gamma$ secretion was observed by BAL T cells from BeS subjects ( $n=8$, data not shown), whereas HLA-DP2 patients $(n=3)$ had discrete populations of Be-induced IFN- $\gamma$-expressing $\mathrm{CD} 4^{+} \mathrm{T}$ cells in BAL, but these cells did not bind the HLA-DP2 reagent (representative example of HLA-DP2- CBD subject is shown in Figure 6F). In CBD patient 8845, $\mathrm{T}$ cells specif- ic to HLA-DP2-chemokine/Be ligands dominated the lung CD4 ${ }^{+}$ $\mathrm{T}$ cell population (Figure $6 \mathrm{G}$ ). While control tetramer staining (HLA-DP2-CLIP) and intracellular IFN- $\gamma$ expression were negative without Be stimulation (Figure $6 \mathrm{G}$ ), $22 \%$ and $25 \%$ of $\mathrm{CD}^{+} \mathrm{T}$ cells from the BAL of patient 8845 bound the HLA-DP2-CCL4/Be and HLA-DP2-CCL3/Be tetramers, respectively. Following 6 hours of Be stimulation, approximately $33 \%$ of the $\mathrm{CD}^{+} \mathrm{T}$ cells in the BAL of this patient expressed IFN- $\gamma$ (Figure 6G). Among this activated population, more than one-third of these cells were specific for the HLA-DP2-chemokine/Be ligand. These data correspond with the large percentage of $\mathrm{T}$ cells expressing the LKGGG CDR3 $\beta$ motif identified by single-cell sequencing in this patient. Furthermore, of the 3 hybridomas studied in this patient, all recognized the CCL4 peptide. However, 1 hybridoma (8845-c3r) did not recognize the CCL3 peptide. Thus, while a large fraction of tetramer-binding $T$ cells will likely be crossreactive to both ligands, our data suggest that some will be specific to only one or the other epitope. A summary of patient tetramer and ICS data is provided in Figure 6, D and E. Overall, CD4 ${ }^{+} \mathrm{T}$ cells specific for either HLA-DP2-CCL/ Be epitope accounted for $5.3 \%$ to $38.5 \%$ of Be-responsive IFN- $\gamma$ expressing $\mathrm{CD}^{+}{ }^{+} \mathrm{T}$ cells in BAL. As noted, patients that exhibited no activated $\mathrm{T}$ cells, as measured by IFN- $\gamma$, had very small or undetectable populations of tetramer-positive cells.

Ex vivo BAL samples from patients 8845 and 8133 were available from a time point 5 years after the initial analysis (Figure 6H). Activation of $\mathrm{CD} 4^{+} \mathrm{T}$ cells as measured by IFN- $\gamma$ expression was still elevated in these subjects, though not to previous levels. Similarly, HLA-DP2-CCL3/Be tetramer binding T cells remained present, though reduced in both patients (Figure 6H). We also compared HLA-DP2-CCL3/Be with HLA-DP2-plexin A/Be tetramer staining (Figure $6 \mathrm{H}$ ). Interestingly, in both patients, the number of cells staining with the CCL3/Be ligand was greater than observed with the HLA-DP2-plexin A/Be tetramer (3.7-fold greater in 8845; 2 -fold in 8133). These data suggested that the specificity to chemokine neoantigens may be significantly greater than the previously described specificity to Be-modified plexin A peptides.

Chemokine/Be-specific CD4 $4^{+} T$ cells traffic to the lungs in TCR retrogenic HLA-DP2 transgenic mice. To investigate the pathogenic potential of $\mathrm{CD}^{+} \mathrm{T}$ cells bearing the LKGGG CDR3 $\beta$ motif, we utilized a model of Be-induced inflammation in HLA-DP2 $\mathrm{Tg}$ C57BL/6 mice $(18,19)$. First, we documented by tetramer staining that mice exposed to $\mathrm{BeO}$ using our standard 21-day sensitization/ boost protocol developed an adaptive immune response to the same ligands as identified in HLA-DP2-expressing CBD patients (Figure 7A). Costaining with both HLA-DP2-CCL/Be tetramers labeled with different fluorochromes showed $\mathrm{CD} 4^{+} \mathrm{T}$ cells with crossreactivity to both ligands, as indicated by the diagonal staining pattern (Figure 7A). In addition, BAL fluid collected at the time of sacrifice had elevated levels of CCL 4 and CCL3 proteins in mice treated with BeO compared with PBS-treated control mice (CCL4, $52.5 \pm 16.2 \mathrm{pg} / \mathrm{mL}$ [mean, STD, BeO treated] vs. $4.4 \pm 4.0 \mathrm{pg} / \mathrm{mL}$ [PBS]; CCL3, $58.0 \pm 19.0$ [BeO] vs. $4.5 \pm 3.3 \mathrm{pg} / \mathrm{mL}$ [PBS]) (Figure $7 \mathrm{~B})$, demonstrating the source of antigen was amplified and available for processing and presentation in the lungs of these mice.

TCR retrogenic mice expressing either the 8845 -c3 TCR or the control DV-13 TCR were generated as previously described (20). Prior to these experiments, we showed that hybridoma 8845-c3 rec- 
A
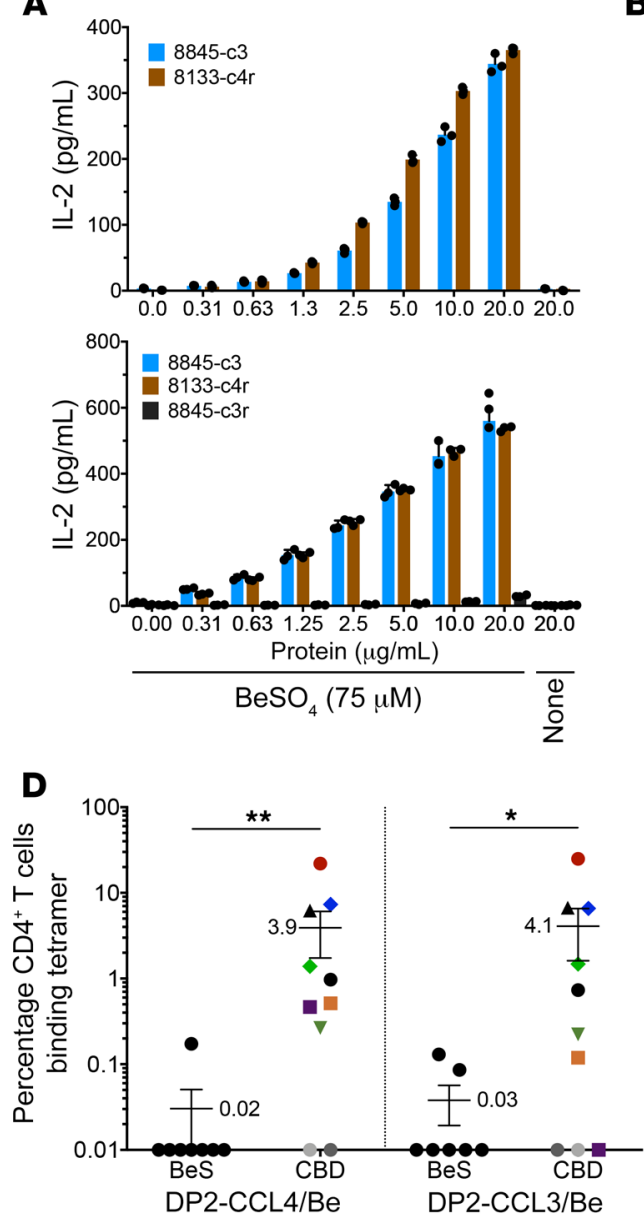

$\mathbf{F}$
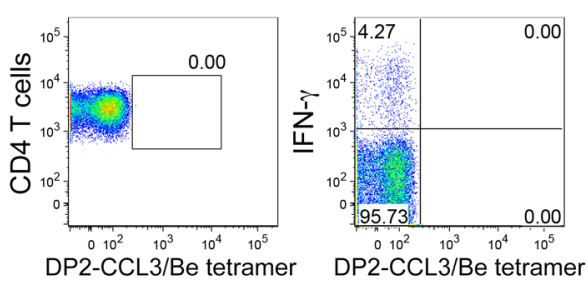

G
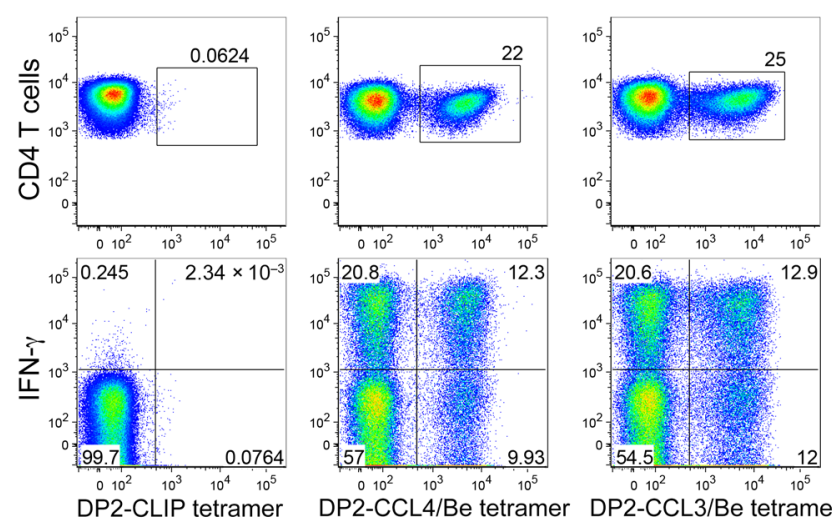

B

E
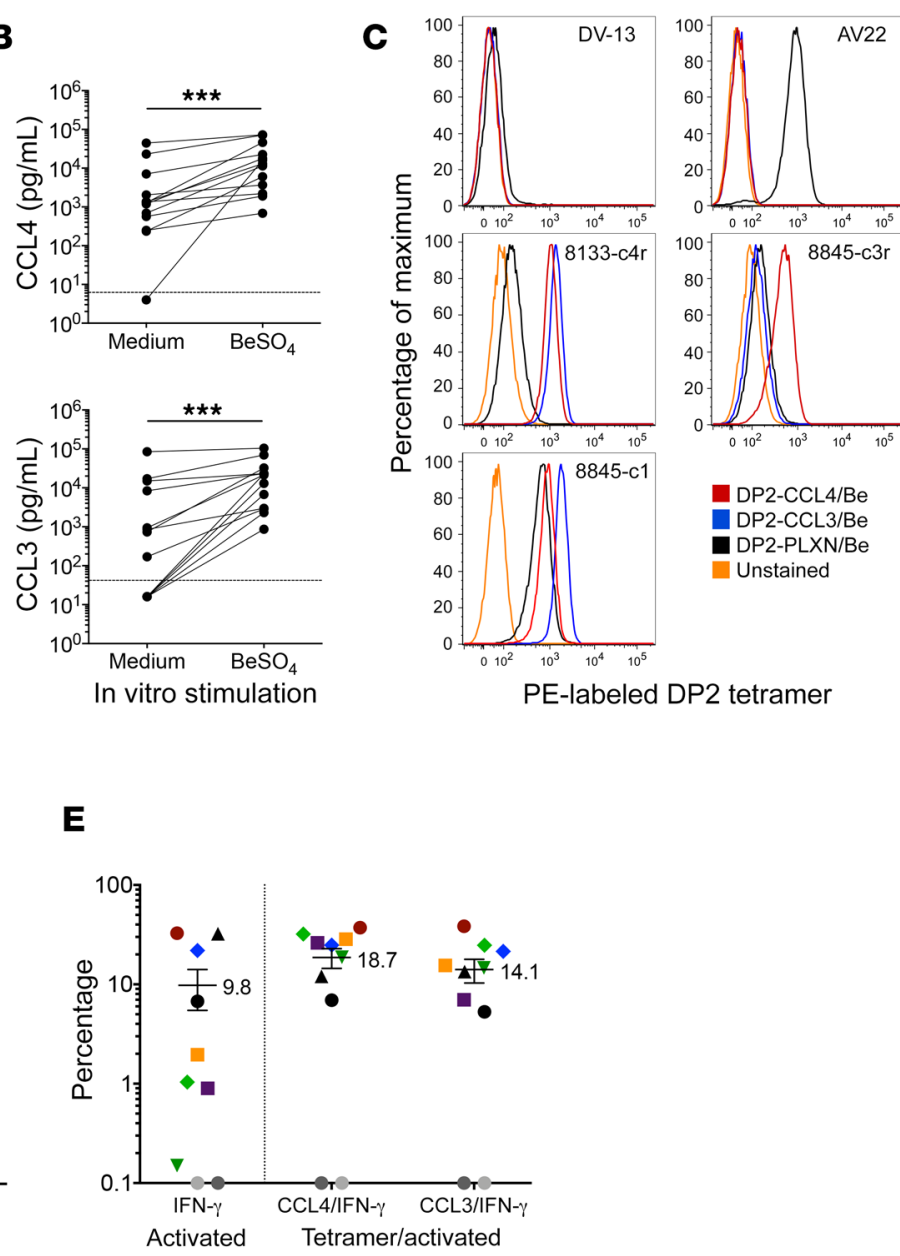

H
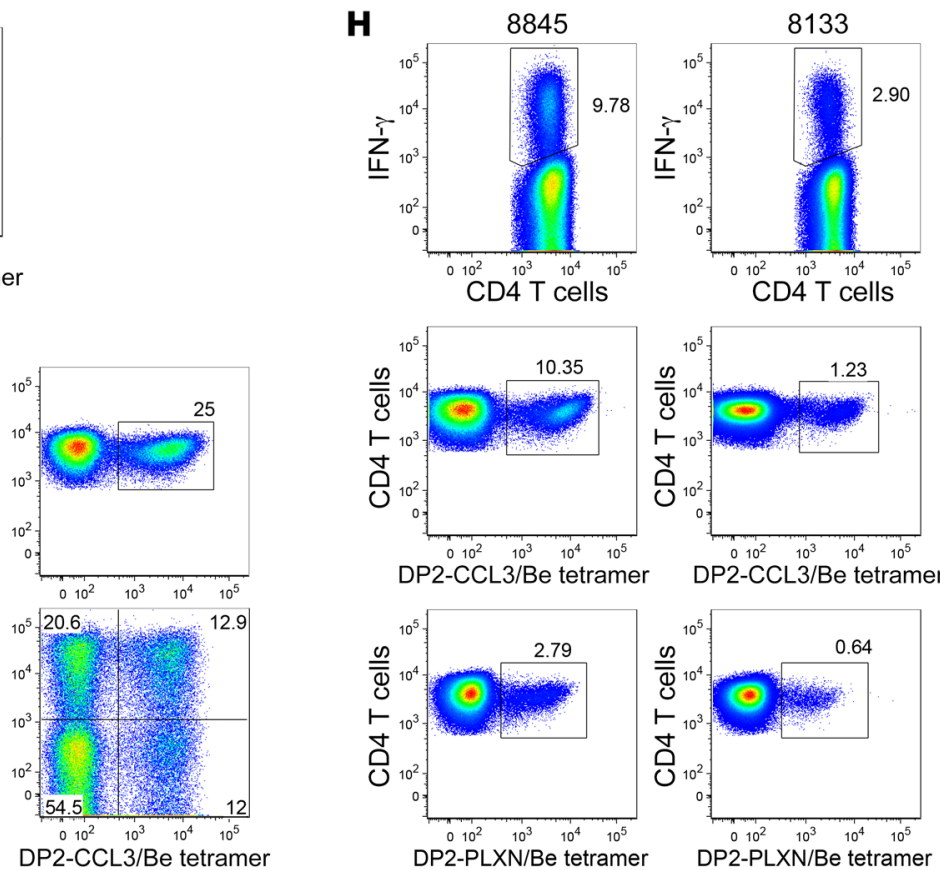
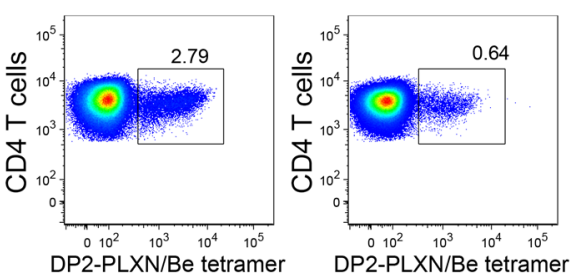
Figure 6. Validation of Be-modified CCL4 and CCL3 as antigenic targets of CD4 $^{+} \mathbf{T}$ cells in CBD. (A) IL-2 response (mean $\pm \mathrm{SD} \mathrm{pg/ml)} \mathrm{of} \mathrm{LKGGG} \mathrm{CDR3} \beta$ hybridomas to recombinant CCL4 (upper) and CCL3 (lower) proteins is displayed. Results are representative of 2 experiments. (B) In vitro Beinduced secretion of CCL4 (upper) and CCL3 (lower) from BAL cells isolated from CBD patients $(n=13)$ is shown. Chemokines were assessed by ELISA in duplicate. Dotted lines represent ELISA limits of detection. Statistical significance was determined using Wilcoxon's rank sum test. ${ }^{* *} P<0.001$. (C) Staining of $\mathrm{T}$ cell hybridomas with $\mathrm{BeSO}_{4}$-saturated $\mathrm{HLA}$-DP2 tetramers. (D) Summary of HLA-DP2 tetramer staining of ex vivo BAL cells from $\operatorname{BeS}(n=7-8)$ and CBD $(n=10)$ subjects. For HLA-DP2+ CBD patients, each subject is represented by a different color/symbol combination. Statistical significance was determined using a Mann-Whitney $U$ test. ${ }^{*} P<0.05$; ${ }^{* *} P<0.01$. (E) Summary of HLA-DP2 tetramer and IFN- $\gamma$ ICS of ex vivo BAL cells from HLA-DP2 ${ }^{+}$CBD patients. Color/symbol combinations used for patients match those in $\mathbf{D}$. Percentages (mean \pm SEM) of $C D 4^{+} T$ cells expressing IFN- $\gamma$ and the fraction of $\mathrm{CD}^{+} T$ cells expressing IFN- $\gamma$ that also bind each tetramer are shown. Gray circles represent patients with no evidence of active disease. (F) Flow cytometric analysis of HLA-DP2 tetramer and intracellular IFN- $\gamma$ staining of ex vivo BAL cells from an HLA-DP2- CBD patient is displayed. (C) Flow cytometric density plots showing the frequency of CD4 ${ }^{+} T$ cells from CBD patient 8845 that bind to HLA-DP2-CLIP (upper left), HLA-DP2-CCL4/Be (upper middle), and HLA-DP2-CCL3/ Be tetramers. Density plots in lower panels depict tetramer staining in relation to IFN- $\gamma$ expression induced in $\mathrm{CD}^{+} \mathrm{T}$ cells after stimulation with $\mathrm{BeSO}_{4}$ (lower left, medium control). (H) Tetramer/ICS staining of ex vivo BAL cells from CBD patients 8845 (left) and 8133 (right) 5 years after single-cell TCR studies and flow cytometric analysis of BAL cells. Cells were stained for intracellular IFN- $\gamma$ (upper panels), HLA-DP2-CCL3/Be (middle panels), and HLA-DP2-PLXN/Be tetramers (lower panels).

ognized the orthologous murine CCL4 $4_{23-32}$ and CCL $3_{22-31}$ peptides, since both differ from their human counterparts by 2 amino acids (Figure 7, C and D). Constructs encoding the 8845-c3 or DV-13 TRA and TRB gene variable domains attached to murine TCR constant regions were established in $R A G^{-/}$HLA-DP2 Tg C57BL/6 mice by retroviral transduction of bone marrow cells (20). After confirming reconstitution of peripheral $\mathrm{T}$ cells (5-6 weeks), mice were exposed to $\mathrm{BeO}$ using a sensitization/boost protocol and sacrificed at day 21. Representative $\mathrm{CD} 4$ immunostaining of lung tissue at low and higher magnification showed that BeO-exposed 8845-c3 TCR retrogenic mice developed $\mathrm{CD}_{4}^{+} \mathrm{T}$ cell infiltrates in a peribronchovascular distribution in contrast to $\mathrm{BeO}$-exposed $\mathrm{DV}-13$ retrogenic mice (Figure $7 \mathrm{E}$ ). Quantitatively, the number of $\mathrm{CD} 4^{+} \mathrm{T}$ cells per $\mathrm{mm}^{2}$ of tissue was significantly increased by approximately 10 -fold in retrogenic mice expressing the 8845-c3 TCR compared with DV-13 retrogenic mice $(P<0.0001)$ (Figure $7 F)$.

The chemokine/Be-specific 8845-c3 $\mathrm{T}$ cells that trafficked to the lung bound tetramer (data not shown) and appeared to be functional and mediate damage. For example, in vitro IFN- $\gamma$ and IL-2 ELISPOT assays measuring splenic T cell responses to $\mathrm{BeSO}_{4}$ showed that BeO-exposed 8845-c3 retrogenic mice had significantly more IL-2- (Figure 7G) and IFN- $\gamma$-producing (Figure 7H) cells than BeO-exposed WT HLA-DP2 Tg B6 mice. Finally, as an indicator of tissue damage, $\mathrm{BeO}$ exposure significantly increased total protein in the BAL fluid of 8845-c3 retrogenic mice compared with PBS-exposed mice $(P<0.05)$ (Figure 7I). Although trends were noted, no significant differences in albumin and T1a were seen in the BAL fluid of $\mathrm{BeO}$-exposed 8845-c3 TCR retrogenic mice and WT HLA-DP2 Tg mice that express a normal, diverse
TCR repertoire. Thus, $\mathrm{CD}^{+}{ }^{+} \mathrm{T}$ cells expressing a single TCR specificity were expanded and recruited to the lung in response to $\mathrm{BeO}$ exposure and mediate some of the disease parameters typically elevated in our murine model of Be-induced disease, suggesting that $\mathrm{CD} 4^{+} \mathrm{T}$ cells bearing this specificity are pathogenic and capable of driving lung inflammation.

LPS exposure amplifies BeO-induced lung inflammation and the number of chemokine/Be-specific CD4 ${ }^{+} T$ cells in the lung of HLADP2 transgenic mice. To determine whether exposure to pathogen-associated molecular patterns (PAMPs) that increase CCL3 and CCL4 expression can modulate the Be-specific adaptive immune response in the lung, we modified our $\mathrm{BeO}$ sensitization/boost protocol to include a single LPS exposure on day 14. A separate cohort of mice were sacrificed on day 15 , and BAL was performed to assess chemokine secretion. As shown in Figure 8A, LPS exposure significantly increased CCL3 and CCL4 in BAL fluid compared with that of mice exposed to $\mathrm{BeO}$ alone $(P<0.001)$. At day 21, lungs were harvested for $\mathrm{CD}^{+} \mathrm{T}$ cell purification, HLADP2-chemokine/Be tetramer staining, and histology. The addition of LPS to the $\mathrm{BeO}$ sensitization/boost protocol substantially increased the number of Be-specific IFN- $\gamma$ - and IL-2-secreting $\mathrm{CD}^{+} \mathrm{T}$ cells in the lung (Figure 8B). Importantly, LPS exposure also increased the percentage of CCL4/Be-specific CD4 $4^{+} \mathrm{T}$ cells in the lung from $8.7 \% \pm 2.4 \%$ after $\mathrm{BeO}$ alone to $20.6 \% \pm 1.1 \%$ $(P<0.01$; Figure 8C) and also increased the absolute number of tetramer positive cells by more than 7-fold (Figure 8C). Representative HLA-DP2-CCL4/Be tetramer staining of murine lung cells is shown in Figure 8D. Conversely, LPS exposure did not affect the percentage of $\mathrm{CD} 4^{+} \mathrm{T}$ cells specific for the HLA-DP2-plexin A/Be epitope (Figure 8E). H\&E staining and quantification of cellular infiltrates confirmed that LPS exposure augmented BeO-induced lung inflammation (Figure 8, F and G). Collectively, these data showed that exposure to a PAMP increased the number of chemokine/Be-specific $\mathrm{CD} 4^{+} \mathrm{T}$ cells in the lung, culminating in an amplified $\mathrm{BeO}$-induced lung inflammation.

\section{Discussion}

CBD is an occupational lung disorder characterized by a well-defined MHCII genetic susceptibility and a known antigenic exposure (21-23). This gene-by-environment interaction results in granulomatous inflammation and the influx of large numbers of Be-specific $\mathrm{CD}_{4}{ }^{+} \mathrm{T}$ cells that are polarized toward a Th-1-type phenotype $(9,11)$. In this study of HLA-DP2-expressing CBD patients, we identified a set of public, disease-associated TCRs expressing a distinct CDR3 $\beta$ motif that recognized Be-modified self-peptides derived from highly related innate immune mediators CCL4 and CCL3. $\mathrm{BeSO}_{4}$-saturated HLA-DP2 tetramers constructed with these chemokine peptides confirmed the widespread nature of this specificity, with $10 \%$ to $40 \%$ of Be-responsive, IFN- $\gamma$-secreting lung $\mathrm{T}$ cells recognizing these neoantigens. Furthermore, T cells in HLA-DP2 ${ }^{+}$TCR retrogenic mice expressing one of these public TCRs expanded and homed to the lung upon Be exposure, suggesting their pathogenic potential. Additional murine studies demonstrated that exposure to a PAMP increased the accumulation of HLA-DP2-CCL/Be-specific CD $4^{+}$ $\mathrm{T}$ cells in the lung and exacerbated Be-induced inflammation. Thus, utilizing an efficient multistep pathway of antigen discov- 
A

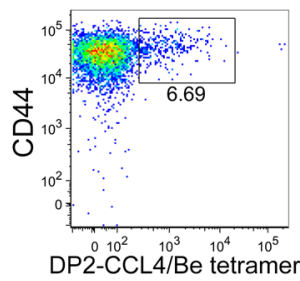

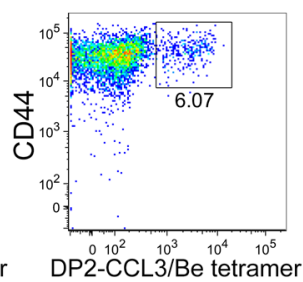

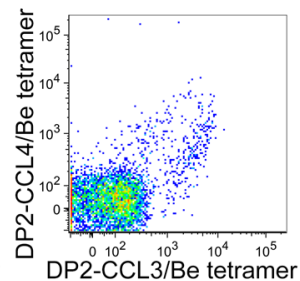

C

\begin{tabular}{lcccccccccc} 
& \multicolumn{1}{c}{ Position } \\
\cline { 2 - 8 } & 1 & 2 & 3 & 4 & 5 & 6 & 7 & 8 & 9 & 10 \\
\hline Human CCL4(23-32) & N & F & $V$ & V & D & Y & Y & E & T & S \\
Mouse CCL4(23-32) & S & F & $V$ & M & D & Y & Y & E & T & S \\
Human CCL3(22-31) & N & F & I & A & D & Y & F & E & T & S \\
Mouse CCL3(22-31) & Q & F & I & V & D & Y & F & E & T & S \\
\hline
\end{tabular}

\section{B}
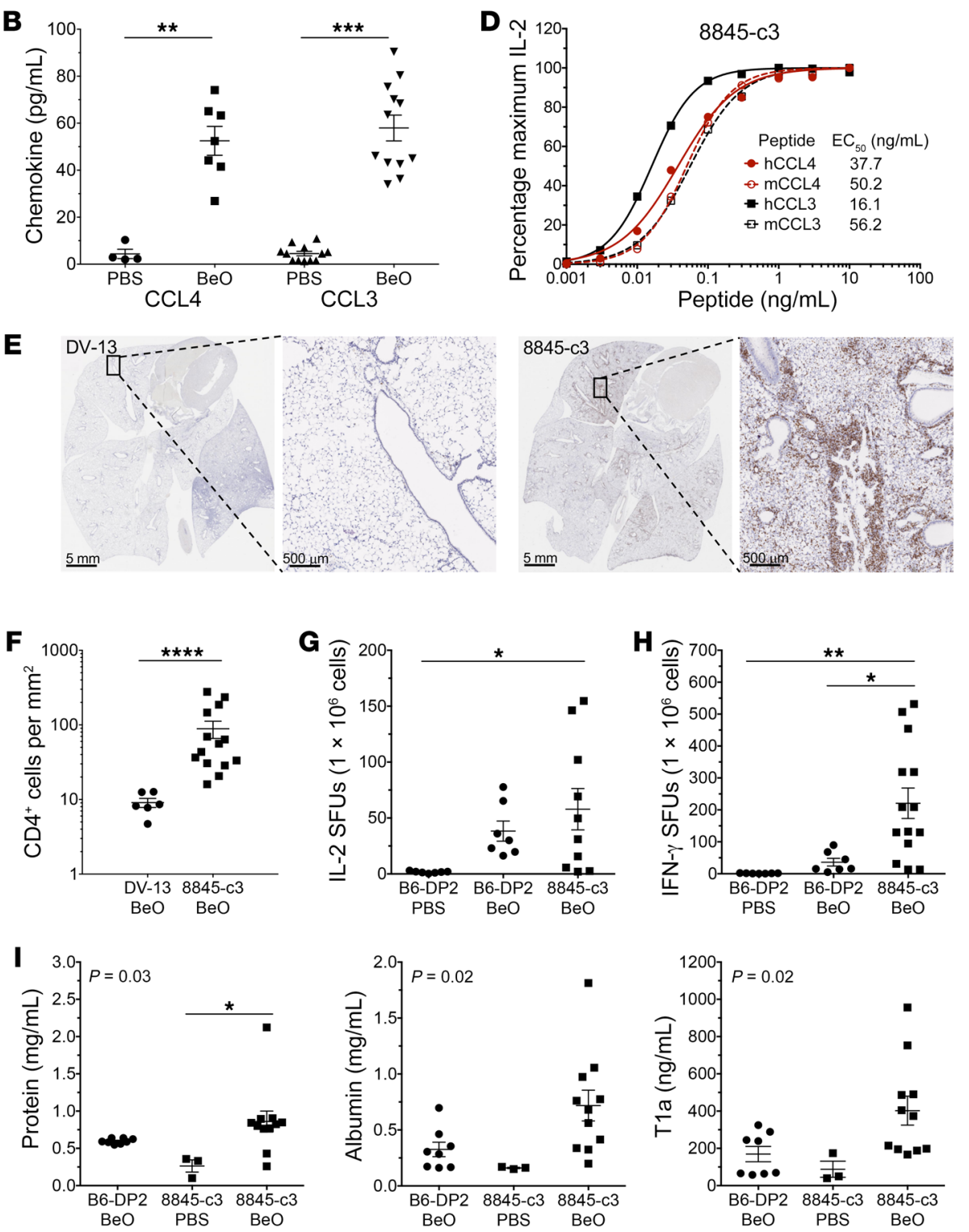

Figure 7. HLA-DP2 transgenic mice and 8845-c3 TCR retrogenic HLA-DP2 mice replicate features of CBD. (A) Flow cytometric density plots illustrating representative HLA-DP2-CCL4/Be (left), HLA-DP2-CCL3/Be (middle), and cotetramer staining (right) of HLA-DP2 transgenic C57BL/6 mouse BAL cells treated with BeO. (B) CCL4 and CCL3 chemokines present in the BAL fluid of BeO-treated C57BL/6 mice. BAL fluid from control (PBS: CCL4, $n=4$; CCL3, $n=$ 12) and treated (BeO: CCL4, $n=7 ;$ CCL3 $n=12$ ) mice was assessed by ELISA. (C and D) Amino acid differences (red) between orthologous human and murine Be-dependent CCL4 and CCL3 epitopes (C) and dose-response curves of hybridoma 8845-C3 (D) comparing its activity to the human and murine peptides in the presence of $\mathrm{BeSO}_{4}$. (E) Representative CD4 staining of lungs from BeO-treated control DV-13 TCR (left panels) and Be-specific 8845-c3 TCR (right panels) retrogenic $R A G^{-1-} \mathrm{B} 6$ mice showing CD4 T cell infiltrates. (F) Quantification of CD4 ${ }^{+} \mathrm{T}$ cells in lung tissue of Be0-treated DV-13 ( $\left.n=6\right)$ and $8845-c 3$ $(n=14)$ retrogenic mice. (G and $\mathbf{H})$ Summary of IL-2- (G) and IFN- $\gamma$-secreting (H) T cells in spleen of PBS-exposed HLA-DP2 Tg mice (IL-2, $n=7$; IFN- $\gamma, n=7)$ and BeO-exposed HLA-DP2 Tg (IL-2, $n=7$; IFN- $\gamma, n=7$ ) and 8845-c3 TCR retrogenic is shown (IL-2, $n=10$; IFN- $\gamma, n=14)$. ELISPOT data are expressed as the mean \pm SEM spot-forming units (SFUs) per $1 \times 10^{6}$ cells. (I) Total protein (left), albumin (middle), and podoplanin (T1a, right) measured by ELISA from BAL fluid of HLA-DP2 Tg C57BL/6 mice treated with PBS $(n=3)$ and BeO $(n=11)$ and BeO-treated 8845-c3 TCR retrogenic mice $(n=8)$. Statistical significance was determined using a Mann-Whitney $U$ test $(\mathbf{B}$ and $\mathbf{F})$ and 1-way ANOVA (G-I). Bars for all data plots represent mean values \pm SEM. ${ }^{*} P<0.05 ;{ }^{* *} P<$ $0.01 ;{ }^{* *} P<0.001 ;{ }^{* * *} P<0.0001$, determined by 1-way ANOVA. Data are pooled from separate experiments. 

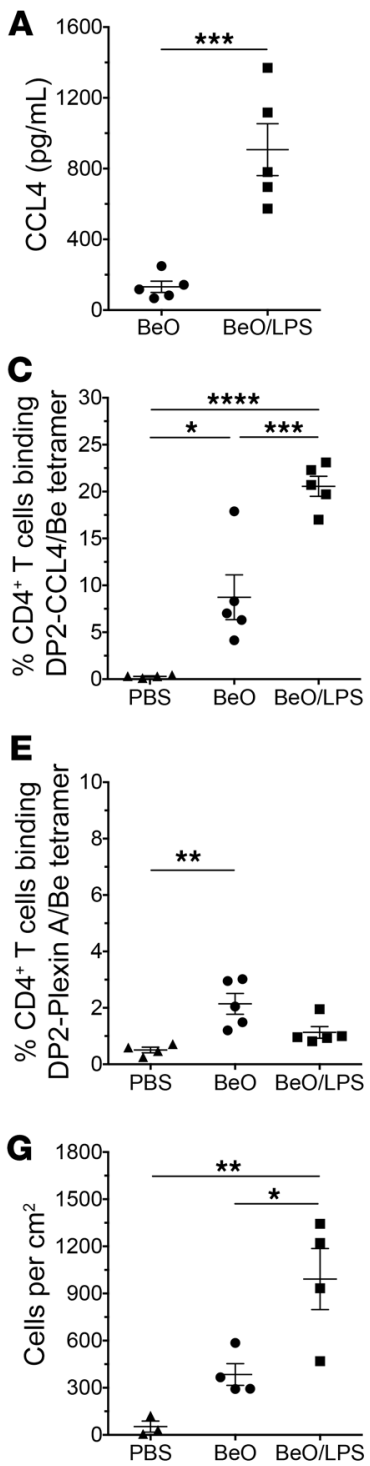
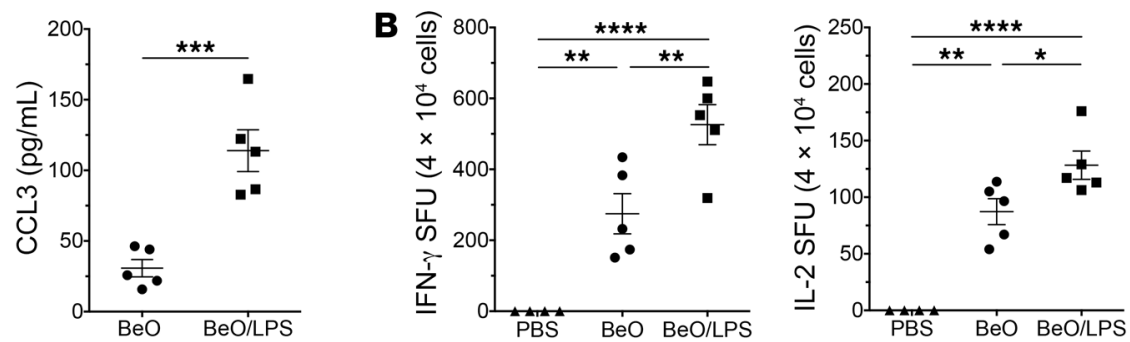

D
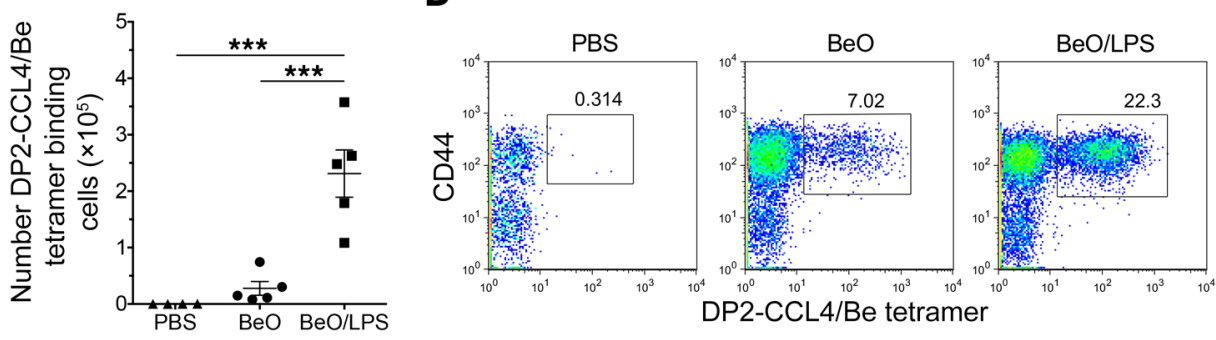

$\mathbf{F}$

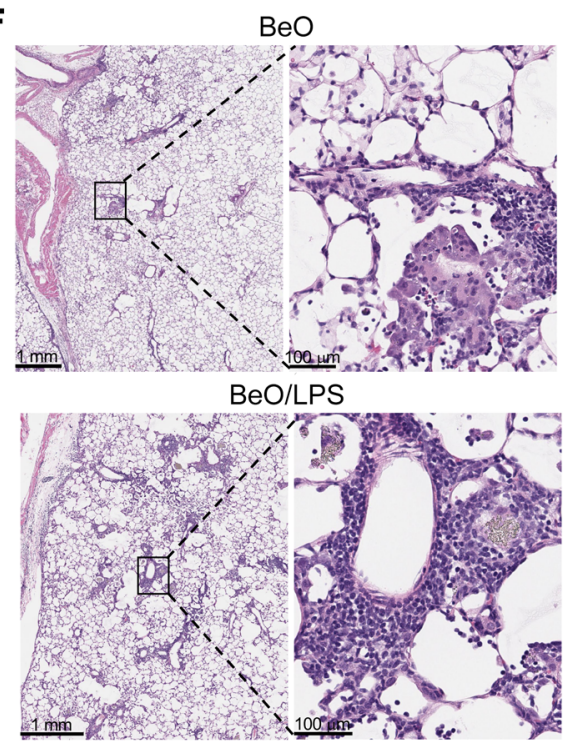

Figure 8. LPS increased chemokine/Be-specific CD4 ${ }^{+}$T cells and inflammation in BeO-exposed HLA-DP2 Tg mice. (A) CCL4 (left) and CCL3 (right) measured by ELISA (pg/ml) in the BAL fluid of FVB/N HLA-DP2 Tg mice. After standard sensitization with BeO, mice were boosted on day 14 with either $\mathrm{BeO}(n=5)$ or BeO plus LPS $(10 \mu \mathrm{g} ; n=5)$ and sacrificed 24 hours later. (B) Frequency of Be-specific CD4+ T cells at day 21 in the lungs of PBS- $(n=4)$, $\mathrm{BeO}-(n=5)$, and BeO/LPS-treated $(n=5)$ HLA-DP2 Tg mice measured by IFN- $\gamma$ (left) and IL-2 (right) ELISPOT is shown. Data are expressed as the mean \pm SEM spot-forming units per $4 \times 10^{4}$ cells. (C) Shown are the frequency (left) and total number (right) of HLA-DP2-CCL4/Be tetramer staining CD4 ${ }^{+} \mathrm{T}$ cells isolated from the lungs of PBS- $(n=4), \mathrm{BeO}(n=5)$, and BeO/LPS-treated mice $(n=5)$ sacrificed on day 21. (D) Flow cytometric density plots illustrating representative HLA-DP2-CCL4/Be tetramer staining of lung cells derived from PBS- (left), BeO- (middle), and BeO/LPS-exposed (right) HLA-DP2 Tg mice sacrificed on day 21. (E) Frequency of HLA-DP2-plexin A/Be tetramer staining CD4 ${ }^{+} T$ cells from the lungs of mice treated as indicated (PBS, $n=4$; BeO, $n=5$; BeO/LPS, $n=5$ ). (F) Representative H\&E staining of lungs from BeO-treated (upper panels) and BeO/LPS-treated (lower panels) mice showing mononuclear cell infiltrates at low and higher magnification. (G) Quantification of mononuclear cells in lung tissue of HLA-DP2 Tg mice treated as indicated (PBS, $n=3$; BeO $n=4$; BeO/LPS, $n=4$ ). Bars for all data plots represent the mean \pm SEM. ${ }^{*} P<0.05 ;{ }^{* *} P<0.01$; ${ }^{* * *} P<0.001$; ${ }^{* * * *} P<0.0001$, Mann-Whitney $U$ test (A) and 1-way ANOVA (B, C, E, and $\mathbf{G})$. Data are representative of 2 separate experiments.

ery, we demonstrated a direct link between Be-induced innate immune production of inflammatory chemokines and oligoclonal expansions of autoreactive $\mathrm{CD} 4^{+} \mathrm{T}$ cells targeting those same chemokines presented as neoantigens in the lung.

The discovery of 2 related chemokines as the source of peptides recognized as Be-modified neoantigens by $\mathrm{CD} 4^{+} \mathrm{T}$ cells has profound implications regarding possible mechanisms of $C B D$ and potentially other exposure-related diseases. CCL3 (macrophage inflammatory protein-1 $\alpha$ ) and CCL4 (macrophage inflammatory protein-1 $\beta$ ) are small chemotactic proinflammatory cytokines that recruit and direct the migration of immune cells to sites of infection or inflammation (24). The cellular sources of CCL3 and CCL4 are broad, and these chemokines exert their influence on an array of different immune cells at multiple points during an immune response. For example, tissue sentinel immune cells, such as mast cells, macrophages, and dendritic cells, release CCL3, CCL4, and other inflammatory molecules when their pattern recognition receptors are engaged (24). These mediators draw innate cells 
(e.g., neutrophils and monocytes) and lymphocytes from blood to rapidly amplify the effector response, and these cell subsets are themselves capable of secreting and responding to CCL3 and CCL4. In essence, both antigen-presenting cells and T cells secrete CCL3 and CCL 4 in the lung and promote enhancement of chemokine and cytokine expression in tissues where antigen-presenting cells and T cells interact.

Relevant to the development of metal hypersensitivity, Be serves not only as an antigenic stimulus, but also as its own adjuvant (25). Macrophages and dendritic cells exposed to Be release inflammatory chemokines and cytokines (26-28), and compared with control subjects, CBD patients upregulate CCL3 expression in blood upon Be exposure (29). In a murine model of CBD, pulmonary exposure to Be induces the release of damage-associated molecular patterns (DAMPs), IL- $1 \alpha$, and TNF- $\alpha$. These cytokines precipitate inflammatory chemokine release in the lung, accompanied by neutrophil infiltration and mobilization of immunogenic dendritic cells from the lung to the lung-draining lymph nodes (30). These early events were required to break peripheral tolerance and allow expansion of $\mathrm{CD}^{+}{ }^{+} \mathrm{T}$ cells reactive to Be-modified self-peptides $(25,30)$. In mice and humans, we showed that CCL3 and CCL4 are secreted within the lung in response to Be and that these proteins are processed by antigen-presenting cells to yield the key stimulatory epitopes recognized by large fractions of Be-specific $\mathrm{CD}^{+} \mathrm{T}$ cells, thus validating that these Be-induced chemokines are also Be-modified targets in the lung.

$\mathrm{Be}$ is considered a persistent antigen, since the metal remains embedded within lung tissue years after workplace exposure has ceased (31). Our findings suggest that continual Be exposure creates a destructive cycle of Be-induced CCL3 and CCL4 secretion by innate immune cells, phagocytosis and processing of these same proteins by antigen-presenting cells, and presentation of Be-modified neoantigens to antigen-specific effector $\mathrm{CD} 4^{+} \mathrm{T}$ cells. Interestingly, any airborne exposure that induces chemokine expression can potentially make these proteins available to antigen-presenting cells for presentation. In this regard, the addition of a single dose of LPS to the BeO sensitization/boost protocol in HLA-DP2 Tg mice increased the number and percentage of CCL4/Be-specific CD $4^{+}$ $\mathrm{T}$ cells as well as mononuclear cellular infiltrates. Although these chemokines mediate multiple innate effects, the mechanism of this enhanced immune response was likely the increased availability of CCL3 and CCL4 as sources of antigen, since a second Bedependent neoantigen (HLA-DP2-Plexin A/Be) was not similarly increased. Importantly, these findings have clinical implications for disease progression in HLA-DP2-expressing CBD patients. For example, bacterial or viral infections in the lung result in the release of PAMPs that activate analogous pathways of innate and adaptive immune activation and augment neoantigen formation. This cascade of events may culminate in the amplification of the HLA-DP2-CCL/Be-specific CD4 ${ }^{+} \mathrm{T}$ cell pool in CBD patients, potentially leading to worsening disease severity. This cycling of activity may explain the exceedingly large accumulations of HLADP2-CCL/Be-specific CD4 ${ }^{+} \mathrm{T}$ cells observed in the BAL of some CBD patients. Many MHCII-peptide tetramer studies that quantify $\mathrm{CD} 4^{+} \mathrm{T}$ cell responses against bacterial, viral, or tumor epitopes are done with blood and require bead enrichment of low-frequency antigen-specific cells (32-34). One study examining CD4 ${ }^{+} \mathrm{T}$ cell responses to Mycobacterium tuberculosis antigens detected large populations of responding cells (35). However, to our knowledge, no studies have documented such a high frequency of antigenspecific $\mathrm{CD}^{+} \mathrm{T}$ cells in the target organ of human beings.

A longstanding problem for the interrogation of antigen specificity in immune-mediated diseases is the requirement of maintaining effector memory $\mathrm{CD} 4^{+} \mathrm{T}$ cell clones in culture for extended periods (36). As an alternative, we determined the expressed $T R A$ and TRB genes directly from individual $\mathrm{T}$ cells so that the paired genes could be transferred to immortalized cell lines for antigen studies. The advent of reliable single-cell assays for ascertaining $\alpha \beta$ TCR pairs has greatly accelerated the process of identifying Be-specific CD4 ${ }^{+} \mathrm{T}$ cells. The RT-PCR assay we employed was robust, capturing both $\alpha$ - and $\beta$-chains in $70 \%$ of the sorted $\mathrm{T}$ cells screened, with complete pairs identified for $54 \%$ of the cells and no evidence that individual $\mathrm{V}$ genes were missed due to inefficient amplification. The success rate of obtaining a positive PCR product was somewhat increased for T cells sorted from in vitroexpanded T cell lines compared with ex vivo BAL cells, suggesting that cellular RNA content might have been the determining factor for whether gene amplification successfully occurred.

Complementing our ability to obtain complete $\alpha \beta$ TCRs, analytical approaches devised to quantitatively measure similarity and cluster TCRs from diverse repertoires also facilitate unbiased antigen discovery. Dash et al. (13) and Glanville et al. (37) examined tetramer-sorted $\mathrm{T}$ cell populations and created metrics to define TCR specificity groups. Application of these algorithms to large-scale diverse TCR repertoire studies provides a rationale for binning and selecting individual $\mathrm{T}$ cells for further investigation. Grouping patients by inferred HLA restriction, such as our focus on HLA-DP2 ${ }^{+}$CBD patients, optimizes this approach, and the methodology must account for predispositions in the unperturbed repertoire shaped by V-J and V-D-J rearrangement processes during $\mathrm{T}$ cell development. Accordingly, nucleotides encoding lysine within the LKGGG CDR3 $\beta$ motif were not typically derived from TRBV, BD, or BJ germline elements, nor was lysine regularly observed at the same position in a reference $\mathrm{T}$ cell population unselected by antigen (13). The high frequency of this amino acid suggested that it was selected by the chemokine peptide/Be neoantigen. In addition, while $T R B V$ usage was somewhat restricted, $T R A V, T R A J, T R B J, C D R 3 \alpha$, and junctional lengths were diverse among the LKGGG CDR3 $\beta$ TCRs. While these findings are in agreement with previous observations regarding $T R$ gene usage of Be-specific CD4 ${ }^{+} \mathrm{T}$ cells $(11,38-40)$, they are in contrast to observations of TCRs identified in pathogen-specific responses that typically rely both on unique CDR3 features and $\mathrm{V}$ and $\mathrm{J}$ gene usage enrichment $(13,37)$. These differences are likely driven by the unique physical and chemical properties of $\mathrm{Be}^{2+}$ that require tetrahedral coordination by acidic amino acids expressed by both the HLA-DP2 $\beta$-chain and peptide (10).

In our previous work defining ligands in $\mathrm{CBD}$, we identified a posttranslational modification entailing specific peptides capturing $\mathrm{Be}^{2+}$ cations to create neoantigens that underlie the adaptive immune response in $\operatorname{CBD}(11,21)$. The relevant characteristic of these peptides that coordinate Be within the HLA-DP2 binding groove is the presence of acidic amino acids located in close proximity to the HLA-DP2 acidic P4 pocket $(10,16)$. Thus, stimulatory 
Be-dependent mimotopes and plexin A peptides included $\mathrm{p} 4 \mathrm{D}$ and p7E $(10,40)$, and absence of either of these amino acids or mutagenesis of glutamic acids at positions 26, 68, or 69 of the HLA-DP2 $\beta$-chain that forms the acidic pocket abrogated the Be-specific response (41). The investigation of the LKGGG CDR3 $\beta$ $\mathrm{T}$ cells using an unbiased approach quickly pointed in the same direction regarding the necessity of properly spaced acidic amino acids. Surprisingly, substituting other combinations of acidic amino acids at p5D and p8E positions (binding HLA-DP2 at P4 and P7) was tolerated by particular TCRs. For example, 8133-c4 recognized all variants at these positions (i.e., D5D8, E5E8, E5D8) despite having strict amino acid requirements at other positions in the peptide. Structural information or high-resolution modeling of individual LKGGG CDR3 $\beta$ TCR footprints over the HLA-DP2chemokine/Be complex would likely allow a dissection of spacing requirements for tetrahedral coordination of the $\mathrm{Be}^{2+}$ cation.

Because of various biologic constraints imposed upon Bedependent epitopes, there is some overlap in the amino acid composition of plexin A and CCL peptides aligned for HLA-DP2 binding. As noted, both include negatively charged aspartic acid and glutamic acid residues at P4 and P7 that represent Be coordination sites. In addition, both utilize bulky hydrophobic amino acids at P1 (phenylalanine) and P6 (phenylalanine or tyrosine) to anchor the peptides within the groove, and finally, alanine scans indicated that LKGGG CDR3 $\beta$ TCRs did not make significant contacts to amino acids on the C-terminus of the peptide; this is similar to the structural data available for the AV22 TCR interacting with the HLA-DP2-mimotope/Be complex (10). Thus, differences between CCL and plexin peptides at P3 (valine/alanine vs. aspartic acid) and P5 (tyrosine vs. leucine) predominantly dictated recognition of these ligands by the TCRs in our study. Hybridoma 8845-c1 appeared to be truly crossreactive, being activated by all WT and variant peptides. Although this TCR CDR3 $\beta$ included a lysine in the appropriate position, we did not anticipate that it would be clustered with the other TCRs because of the extended length of its CDR3 $\beta$. The length of this diversity region may be what enables this receptor to interact with different HLA-DP2-peptide/Be complexes.

Biometrical analysis of PSL screening data is based on a model of independent contribution of individual amino acids to epitope recognition by $\mathrm{T}$ cells and has proven to be a powerful tool for assigning stimulatory potentials to all peptides available in any protein database $(42,43)$. Because we had a cluster of TCRs with presumed similar antigen specificity, separate biometrical analyses were completed and results from each TCR were merged. This process created a single list of potential stimulatory peptides representing not the best candidates for any single TCR, but rather the most likely to stimulate all the TCRs. Having multiple TCRs for screening of candidate stimulatory peptides also aided the process of honing in on the true Be-dependent epitopes. At least one hybridoma responded to 19 of the 100 synthesized peptides, and some individual hybridomas responded to more than 10 different peptides. However, only the CCL4 peptide activated all TCRs in the presence of $\mathrm{Be}$, and the highly related CCL3 peptide activated 8 of 9 total hybridomas. In this context, those TCRs with the most restricted requirements for peptide recognition (see Figure $3 \mathrm{~A}$ sequence logos of 8133-c4 and 8845-c3r compared with 8133-c4r and $8845-\mathrm{c} 3$ ) that still give detectable signals during PSL screens may be the most useful for discriminating among large sets of peptides. Collectively, combining single-cell TCR analysis of ex vivo $\mathrm{T}$ cells derived from the organ of interest and biometrical analysis of PSL data provided an efficient pathway for antigen discovery and should enable the delineation of disease-relevant epitopes in other immune-mediated disorders of unknown etiology, such as sarcoidosis and rheumatoid arthritis.

In conclusion, we have identified chemokines involved not only in innate inflammatory cell recruitment, but also encoding previously undescribed Be-modified self-peptides that bind to HLA-DP2 and represent a dominant CD4 ${ }^{+} \mathrm{T}$ cell epitope in CBD. The public $\mathrm{T}$ cells that respond to these related ligands are expanded and persist in the lungs of CBD patients and likely contribute to continued inflammation and disease progression. Among antigenic drivers of inflammation, Be is unique in its ability to persist within the lung as well as to function as both an adjuvant and antigen, creating a "perfect storm" of innate immune activation and a continual supply of CCL3 and CCL4 to drive recruitment of neoantigen-specific $\mathrm{CD} 4^{+} \mathrm{T}$ cells to the lungs of $\mathrm{HLA}-\mathrm{DP} 2^{+} \mathrm{CBD}$ patients.

\section{Methods}

Study population. CBD was diagnosed using previously defined criteria, including a history of Be exposure, the presence of granulomatous inflammation, and a positive proliferative response of PBMC or BAL $\mathrm{T}$ cells to $\mathrm{BeSO}_{4}$ in vitro $(44,45)$. Demographics of the CBD patients are shown in Supplemental Table 5. The diagnosis of BeS was established based on a history of Be exposure, an abnormal blood BeLPT on 2 occasions, and the absence of granulomatous inflammation or other abnormalities on lung biopsy $(46,47)$. Unless noted, patients chosen for study expressed HLA-DP2 (HLA-DPB1*02:01/HLA-DPA1*01:03). For several subjects, BAL-derived T cell lines stimulated with $\mathrm{BeSO}_{4}$ were generated $(41,48)$.

Single-cell PCR of T cell receptor genes. Cryopreserved ex vivo BAL cells were placed in culture to adhere alveolar macrophages. Collected nonadherent ex vivo cells or thawed T cell lines from CBD patients were cultured overnight in complete RPMI-1640 supplemented with $40 \mathrm{U} / \mathrm{mL}$ rIL-2. Cells were sorted based on $\mathrm{CD}^{+}, \mathrm{CD}^{+}, \mathrm{CD}^{-}$, and DAPI ${ }^{-}$expression using a Moflow Astrios EX flow cytometer (Beckman Coulter) directly into RT buffer in Nunc 96-well polypropylene plates with 1 empty well as a negative control. cDNA synthesis and PCR were performed to amplify the TRA and TRB genes expressed by each $\mathrm{T}$ cell and to incorporate barcodes on both ends of each PCR product, as previously described (49).

A sample of each PCR reaction was run on an agarose gel to determine the presence of a PCR product. PCR products from all wells were pooled, concentrated (Nucleospin Kit, Takara Bio), gel purified (QIAquick Gel Extraction Kit, QIAGEN), and sequenced using $2 \times 250$ paired end reads on an Illumina MiSeq System. Sequencing data were groomed, processed, and barcode split using the web-based Galaxy platform (https://usegalaxy.org). TCR sequences were deposited in the NCBI's Sequence Read Archive (SRA BioProject PRJNA689822).

Analysis of expressed TCRs from the BAL of CBD patients. Data were analyzed using an in-house software pipeline to identify TRA and TRB gene usage (IMGT nomenclature) and extract CDR3 sequences for each $\mathrm{T}$ cell. To be included in subsequent analyses, a minimum of 200 identical reads of a functional $T R$ gene must have been obtained for a $\mathrm{T}$ cell and those reads must have made up at least $10 \%$ of the total 
reads acquired. Previously developed analytical tools were employed to quantitatively assess TR gene segment usage, TCR distance measurements (TCRdist), clustering, and CDR3 motifs to the sequencing data (13). Baseline values for comparing $T R$ gene or CDR3 amino acid frequencies were obtained from a background, nonepitope selected TCR repertoire from a combination of high-throughput TCR repertoire profiling experiments (13).

PSLs, scoring matrices and database searches. $\mathrm{T}$ cell hybridomas expressing selected TCRs were generated and screened for responses against unbiased and biased decapeptide PSLs, as previously described $(11,14,15)$ and as detailed in the Supplemental Methods. Biometrical analysis of the biased PSL screening results (i.e., generation of scoring matrices and searching of a human protein database) is detailed in the Supplemental Methods and is as previously described (14, 43).

Tetramer staining and dual intracellular IFN- $\gamma /$ tetramer assay. Be-saturated MHCII tetramers with covalently attached peptides were generated using a baculovirus expression system $(11,50)$, and the HLA-DP2-CLIP tetramer was provided by the NIH Tetramer Core Facility at Emory University (Atlanta, Georgia, USA). Tetramer and ICS are detailed in the Supplemental Methods.

HLA-DP2 Tg and TCR retrogenic HLA-DP2 Tg mice. C57BL/6 and FVB/N HLA-DP2 transgenic mice were housed and bred at the University of Colorado Biological Resource Center. C57BL/6 RAG ${ }^{-/-}$mice were purchased from The Jackson Laboratory and bred to express HLA-DP2. Generation of TCR retrogenic mice $(20,51)$ and all experiments conducted with different mouse strains were as previously described $(18,19)$ and as detailed in the Supplemental Methods.

Statistics. Statistical tests were performed using GraphPad Prism software. Data were presented as mean or median \pm SD or SEM, as stated in figure legends. Statistical significance tests for parametric or nonparametric tests were determined as indicated. $P$ values of less than 0.05 were considered statistically significant.
Study approval. Each CBD and BeS patient provided informed consent prior to inclusion in the study, and the protocol was approved by the Human Subject Institutional Review Boards at the University of Colorado Anschutz Medical Campus and National Jewish Health. For mouse studies, all experiments were approved by the Institutional Animal Care and Use Committee of the University of Colorado Anschutz Medical Campus in accordance with NIH guidelines.

\section{Author contributions}

MTF and APF conceptualized the study and wrote the original manuscript. LAM provided patient samples and data analysis. MTF and ANT performed the majority of experiments, with contributions from AKM, DGM, SMA, and LL. LGL and MN provided expertise in single-cell sequencing. JCC, PGT, MTF, and APF analyzed and interpreted TCR data. RGS and CP provided peptide libraries and data analysis and assisted in experimental design with MTF and APF. FC and JWK generated tetramer reagents. All authors reviewed and edited the manuscript.

\section{Acknowledgments}

The authors thank the Flow Cytometry Shared Resource and the Genomics and Microarray Shared Resource at the University of Colorado Cancer Center. The authors also thank the Santiago lab for use of their PCR machines and Todd Egan for assistance with bioinformatic processing of single-cell sequencing data. This work was supported by NIH grants (HL62410, HL152756, HL102245, and ESO25534 to APF).

Address correspondence to: Andrew P. Fontenot, University of Colorado Anschutz Medical Campus, Mail Stop B164, 12700 E. 19th Ave., Aurora, Colorado 80045, USA. Phone: 303.724.7192; Email: andrew.fontenot@cuanschutz.edu.
1. Dendrou CA, et al. HLA variation and disease. Nat Rev Immunol. 2018;18(5):325-339.

2. Jurewicz MM, Stern LJ. Class II MHC antigen processing in immune tolerance and inflammation. Immunogenetics. 2019;71(3):171-187.

3. Vizcaino JA, et al. The Human Immunopeptidome Project: a roadmap to predict and treat immune diseases. Mol Cell Proteomics. 2020;19(1):31-49.

4. Delong T, et al. Pathogenic CD4 T cells in type 1 diabetes recognize epitopes formed by peptide fusion. Science. 2016;351(6274):711-714.

5. Faridi P, et al. A subset of HLA-I peptides are not genomically templated: Evidence for cis- and trans-spliced peptide ligands. Sci Immunol. 2018;3(28):eaar3947.

6. Liepe J, et al. A large fraction of HLA class I ligands are proteasome-generated spliced peptides. Science. 2016;354(6310):354-358.

7. Zarnitsyna VI, et al. Estimating the diversity, completeness, and cross-reactivity of the T cell repertoire. Front Immunol. 2013;4:485.

8. Balmes JR, et al. An official American Thoracic Society statement: diagnosis and management of beryllium sensitivity and chronic beryllium disease. Am J Respir Crit Care Med. 2014;190(10):e34-e59.

9. Fontenot AP, et al. Target organ localiza- tion of memory CD4(+) T cells in patients with chronic beryllium disease. JClin Invest. 2002;110(10):1473-1482.

10. Clayton GM, et al. Structural basis of chronic beryllium disease: linking allergic hypersensitivity and autoimmunity. Cell. 2014;158(1):132-142.

11. Falta MT, et al. Identification of berylliumdependent peptides recognized by CD4+ T cells in chronic beryllium disease. J Exp Med. 2013;210(7):1403-1418.

12. Marrack P, Kappler JW. Do MHCII-presented neoantigens drive type 1 diabetes and other autoimmune diseases? Cold Spring Harb Perspect Med. 2012;2(9):a007765.

13. Dash P, et al. Quantifiable predictive features define epitope-specific $\mathrm{T}$ cell receptor repertoires. Nature. 2017;547(7661):89-93.

14. Hemmer B, et al. Identification of candidate T-cell epitopes and molecular mimics in chronic Lyme disease. Nat Med.1999;5(12):1375-1382.

15. Pinilla C, et al. Investigation of antigen-antibody interactions using a soluble, non-support-bound synthetic decapeptide library composed of four trillion $\left(4 \times 10^{12}\right)$ sequences. Biochem J. 1994;301(3):847-853.

16. Dai S, et al. Crystal structure of HLA-DP2 and implications for chronic beryllium disease. Proc Natl Acad Sci U S A . 2010;107(16):7425-7430.
17. Sidney J, et al. Five HLA-DP molecules frequently expressed in the worldwide human population share a common HLA supertypic binding specificity. J Immunol. 2010;184(5):2492-2503.

18. Atif SM, et al. Protective role of B cells in sterile particulate-induced lung injury. JCI Insight. 2019;5:125494.

19. Mack DG, et al. Regulatory T cells modulate granulomatous inflammation in an HLA-DP2 transgenic murine model of berylliuminduced disease. Proc Natl Acad Sci US A. 2014;111(23):8553-8558.

20. Bettini ML, et al. Generation of T cell receptorretrogenic mice: improved retroviralmediated stem cell gene transfer. Nat Protoc. 2013;8(10):1837-1840.

21. Fontenot AP, et al. Beryllium-induced hypersensitivity: genetic susceptibility and neoantigen generation. JImmunol. 2016;196(1):22-27.

22. Fontenot AP, Maier LA. Genetic susceptibility and immune-mediated destruction in beryllium-induced disease. Trends Immunol. 2005;26(10):543-549.

23. McKee AS, Fontenot AP. Interplay of innate and adaptive immunity in metal-induced hypersensitivity. Curr Opin Immunol. 2016;42:25-30.

24. Sokol CL, Luster AD. The chemokine system in innate immunity. Cold Spring Harb Perspect Biol. 
2015;7(5):a016303.

25. McKee AS, et al. MyD88 dependence of beryllium-induced dendritic cell trafficking and $\mathrm{CD}^{+}{ }^{+} \mathrm{T}$-cell priming. Mucosal Immunol. 2015;8(6):1237-1247.

26. Amicosante M, et al. HLA-DP-unrestricted TNF-alpha release in beryllium-stimulated peripheral blood mononuclear cells. Eur Respir J. 2002;20(5):1174-1178.

27. Hong-Geller E, et al. Chemokine regulation in response to beryllium exposure in human peripheral blood mononuclear and dendritic cells. Toxicology. 2006;218(2-3):216-228.

28. Sawyer RT, et al. Beryllium-stimulated apoptosis in macrophage cell lines. Toxicology. 2000;149(2-3):129-142.

29. Li L, et al. Beryllium-induced lung disease exhibits expression profiles similar to sarcoidosis. Eur Respir J. 2016;47(6):1797-1808.

30. Wade MF, et al. TLR9 and IL-1R1 promote mobilization of pulmonary dendritic cells during beryllium sensitization. J Immunol. 2018;201(8):2232-2243.

31. Sawyer RT, et al. Secondary ion mass spectroscopy demonstrates retention of beryllium in chronic beryllium disease granulomas. JOccup Environ Med. 2005;47(12):1218-1226.

32. Moon JJ, et al. Tracking epitope-specific T cells. Nat Protoc. 2009;4(4):565-581.

33. Nepom GT. MHC class II tetramers. JImmunol. 2012; 188(6):2477-2482.

34. Vollers SS, Stern LJ. Class II major histocompatibility complex tetramer staining: prog- ress, problems, and prospects. Immunology. 2008;123(3):305-313.

35. Hohn H, et al. MHC class II tetramer guided detection of Mycobacterium tuberculosis-specific $\mathrm{CD} 4+\mathrm{T}$ cells in peripheral blood from patients with pulmonary tuberculosis. Scand J Immunol. 2007;65(5):467-478.

36. Fontenot AP, et al. Frequency of berylliumspecific, central memory CD4+ T cells in blood determines proliferative response. J Clin Invest. 2005;115(10):2886-2893.

37. Glanville J, et al. Identifying specificity groups in the T cell receptor repertoire. Nature. 2017;547(7661):94-98.

38. Bowerman NA, et al. Mutagenesis of beryllium-specific TCRs suggests an unusual binding topology for antigen recognition. J Immunol. 2011;187(7):3694-3703.

39. Bowerman NA, et al. Identification of multiple public TCR repertoires in chronic beryllium disease. J Immunol. 2014;192(10):4571-4580.

40. Falta MT, et al. Metal-specific CD4+ T-cell responses induced by beryllium exposure in HLA-DP2 transgenic mice. Mucosal Immunol. 2016;9(1):218-228.

41. Bill JR, et al. Beryllium presentation to CD4+ T cells is dependent on a single amino acid residue of the MHC class II beta-chain. J Immunol. 2005;175(10):7029-7037.

42. Sospedra M, et al. Recognition of conserved amino acid motifs of common viruses and its role in autoimmunity. PLoS Pathog. 2005;1(4):e41.

43. Zhao Y, et al. Combinatorial peptide libraries and biometric score matrices permit the quantitative analysis of specific and degenerate interactions between clonotypic TCR and MHC peptide ligands. J Immunol. 2001;167(4):2130-2141.

44. Newman LS, et al. Pathologic and immunologic alterations in early stages of beryllium disease: re-examination of disease definition and natural history. Am Rev Respir Dis. 1989;139(6):1479-1486.

45. Rossman MD, et al. Proliferative response of bronchoalveolar lymphocytes to beryllium. A test for chronic beryllium disease. Ann Intern Med. 1988;108(5):687-693.

46. Newman LS. Significance of the blood beryllium lymphocyte proliferation test. Environ Health Perspect. 1996;104(Suppl 5):953-956.

47. Mroz MM, et al. Reexamination of the blood lymphocyte transformation test in the diagnosis of chronic beryllium disease. J Allergy Clin Immunol. 1991;88(1):54-60.

48. Fontenot AP, et al. Beryllium presentation to CD4+ T cells underlies disease-susceptibility HLA-DP alleles in chronic beryllium disease. Proc Natl Acad Sci U S A. 2000;97(23):12717-12722.

49. Michels AW, et al. Islet-Derived CD4 T cells targeting proinsulin in human autoimmune diabetes. Diabetes. 2017;66(3):722-734.

50. Crawford F, et al. Detection of antigen-specific $\mathrm{T}$ cells with multivalent soluble class II MHC covalent peptide complexes. Immunity. 1998;8(6):675-682.

51. Holst J, et al. Generation of T-cell receptor retrogenic mice. Nat Protoc. 2006;1(1):406-417. 